\title{
Effect of Atorvastatin (ATOR) on the Cardiac Muscle Fibres in Hyperlipidemic Adult Male Albino Rats (Structural and Biochemical Study) Ezz-Eldin E Abdallah $^{(*)}$, Alsaid A Abd-Elhady ${ }^{(* *)}$,Magdy Y Elsaid ${ }^{(* * *)}$ \\ Histology ${ }^{(*)}$, Anatomy ${ }^{(* *)}$ and Physiology ${ }^{(* * *)}$ Departments \\ Faculty of Medicine-Damietta- Al-Azhar University
}

\section{ASBTRACT}

Background:Atorvastatin (ATOR) used as a cholesterol-lowering drug. Ator is one of statins widely used, commercially available and increasingly used day by day.Few studies and limited data were existed on atorvastatin induced histological and ultrastructural changes in the cardiac muscle fibres. The present study was conducted to evaluate the potential toxicity of the human equivalent therapeutic doses of Atorvastatin on cardiac muscle fibres in induced hyperlipidemic adult male albino rats. Material and Methods: thirty adult male albino rats were used in this study, the rats used weighing $(150 \pm 10) \mathrm{gm}$. Rats were divided into groups, Group one (5 rats), served as the control group (C) .Other twenty five rats were subjected to high fat diet (25\% fat and $2 \%$ cholesterol) for 3 weeksand thenthey subdivided into three groups.Group two (five rats) was considered as a hyperlipidemic group $(\mathbf{H})$.Group three, treated group one (T1) (ten rats) received atorvastatin(Ator $20 \mathrm{mg})(1.5 \mathrm{mg} / \mathrm{day} / \mathrm{rat})$ for three weeks, by the end the third week five rats weresacrificed and other five rats were considered as the recovery group one (R1), they received normal diet only for another three weeks. Group four, treated group two (T2) (ten rats) received Atorvastatin (Ator $20 \mathrm{mg})(1.5 \mathrm{mg} / \mathrm{day} / \mathrm{rat})$ for other six weeks , by the end of the $6^{\text {th }}$ week five rats weresacrificed and other five rats were considered as the recovery group two $(\mathbf{R 2})$, they received only normal diet for another six weeks. At the end of each step of the experiment, rats were sacrificed and specimens of cardiac muscle fibres prepared by different methods for light and electron microscopic studies. Also blood sampling was obtained forthebiochemical study.Results: the histological data obtained from the different groups showed many changes, in hyperlipidemic group $(\mathbf{H})$ indistinct and distorted striation and separation of cardiac muscle fibres, ,vesicular nuclei, presence of myofibroblasts with congested and dilated blood vessels, loss of some nuclei and condensation of nuclear chromatin with normal mitochondria were detected. Treated group one (T1) showed that cardiac muscle fibres restored their striation with separated and splitted myofibres and pyknotic nuclei, increased myofibrobasts ,lymphocytic infiltration, congested blood vessels and mitochondrial vacuolation with disorganization of their cristae. In treated group two (T2) patchy loss of striation was observed with splitted cardiac muscle fibres ,pyknotic nuclei of cardiomyocytes, margination of nuclear chromatin ,congested blood vessels, lymphocytic infiltration, swelling of mitochondria with disorganization of their cristae. In recovery group one (R1) cardiac muscle fibres restored their striation. In recovery group two (R2) cardiac muscle fibres restored their normal architecture. The statistically evaluated data showed a significant difference in parentage of collagen and optical density of PAS + ve materials in the different groups when compared to the control group specially in T2.The laboratory evaluated data showedsignificant increase inlevels of cholesterol (C), triglycerides (TG), low density lipoprotein- cholesterol (LDL-C), Very low density lipoprotein (vLDL), Atherogenic index(AI) and decrease in high density lipoprotein- cholesterol (HDL-C) between the control and the hyperlipidemic group. Administration of ATOR to the treated groups induce significant decrease inlevels of C, TG, LDL-C , (vLDL), (AI) and increase of HDL-C when compared with the hyperlipidemic group .Also there were significant decreasein levels of $\mathrm{C}$, TG, LDL-C and increase of HDL-Cin recovery groups when compared with the hyperlipidemic group. Conclusion: administration of ATOR for long time led to adverse degenerative effects on structure of the cardiac muscle fibres, although it has a potent lowering effect on lipid profile in hyperlipidemia.

Key words:Atorvastatin (ATOR), Cardiac muscle fibers, Induced hyperlipidemia , Adult male albino rats. 


\section{INTRODUCTION}

Different studies on the experimental animals had clearly demonstrated the common risk factor for cardiovascular diseases in case of hyperlipidemia. ${ }^{[1]}$

High blood cholesterol and hyperlipidemiaare major risk factors. Epidemiological studies have shown that elevation of serum total cholesterol ,LDL-cholesterol, triglycerides, fibrinogen and platelets count aremajor risk factors for cardiovascular disease (CVD)whichlead to morbidity and mortality in adult human being in different sites of the world even in the developing countries. ${ }^{[2]}$

Treatment of hypercholesterolemia may reduce the risk or development of cardiovascular disease. ${ }^{[3]}$

Various treatments for high blood cholesterol are available. Medication, patient education, dietary changes and exercise are all recommended treatments for hyperlipidemia. ${ }^{[4]}$

Statins (3-hydroxy-3-methylglutaryl coenzyme A (HMG-CoA) reductase inhibitors) are the primary class of medication used to lower serum cholesterol concentration. The most commonly prescribed, atorvastatin, commonly known as Lipitor ${ }^{\left[{ }^{[5]}\right.}$ From 1996 to 2012, atorvastatin became the world's best-selling drug. ${ }^{4}$ Atorvastatin is made in tablet form by generic drug makers under various brand names. ${ }^{[6]}$ In Egypt the drug has a different marketing name, the most common known as Ator.

Atorvastatin (member of lipophilic statins) is classified as a drug that has concentration-dependent adverse effect on muscle cell viability and promote cell disruption via proteolysis and apoptosis. ${ }^{[7]}$

Hypolipidemic drugs cause potentially serious side effects and include digestive disturbances, nausea and vomiting. ${ }^{[8]}$

Using of atorvastatin safely requires regular monitoring for side effects. Atorvastatin has the potential to cause adverse events which can result in muscular inflammation (myositis). ${ }^{[9]}$ In the lastfew years there has been an increased interest regarding the side effects of statins specially atorvastatin. ${ }^{[10]}$

Skeletal muscle side effects that are associated with atorvastatin-use involve muscle cramping, soreness, fatigue, weaknessand, in rare cases, rapid muscle breakdown that can lead to death ( Rhabdomyolysis). ${ }^{[11]}$

Atorvastatin (ATOR) is known to cause skeletal muscle side effects, but it is currently unclear if it also affects the structure of cardiac muscle fibres or not.

\section{MATERIALS AND METHODS}

Thirty adult male albino rats were used in this study; rats used weighing $(150 \pm 10) \mathrm{gm}$. They were left for two weeks in the laboratory room before any experimental interference for acclimatization.All rats were kept under normal conditions of light, temperature and relative humidity, and provided with normal diet pellets and drinking tap water during the whole experimental period.

Following that, rats were divided into the experimental groups. Group one (five rats) was considered as a control group (C).

Other twenty five rats were subjected to high fat diet (HFD) (25\% fat and 2\% cholesterol) for 3 weeks. ${ }^{[12]}$ After induction of hyperlipidemia the rats weighted about (200 \pm 10)gm . Then they were subdivided into three groups.Group two (five rats) was considered as a hyperlipidemic group(H).

Before start the treatment with atorvastatin (Ator 20mg / tablet)(Product of EPICO company ), it was dissolved in distilled water and given orally by a gastric tube . ${ }^{[13]}$ Atorvastatinhuman dosage about $80 \mathrm{mg}$, which equal about $7.2 \mathrm{mg} / \mathrm{kg}$ in rats. [14] ${ }^{\text {and[15] }}$ Sothe daily single oral dose in this study is about $1.5 \mathrm{mg} /$ day / rat.

Group three, treated groupone (T1) (ten rats) were similar to the second group,but received atorvastatin (Ator $20 \mathrm{mg})(1.5 \mathrm{mg} /$ day / rat)for three weeks orally. At the end the third week five rats weresacrificed and other five rats were left without any other treatment andconsidered as the recovery group(R1)and received normal diet only for another three weeks.

Animals of group four (Treated grouptwo,T2) (ten rats) were similar to the second group ,but they received atorvastatin (Ator 20mg) (1.5 mg / day / rat) for six weeks orally. At the end of the sixth week five rats were scarified and other five rats were left without any other treatment andconsidered as the recovery group two(R2)and received normal diet only for another six weeks. 
At the end of each step of the experiment, all animals were anaesthetized by ether inhalation, sacrificedand small pieces of heartswere extracted and fixed in 10\% neutral buffered formol for 48 hours and prepared for paraffin sections. Another piece of specimens were fixed in glutaraldehyde for $\mathrm{E} / \mathrm{M}$ technique.

\section{Parameters of the study}

1- Histological study:

Paraffin sections (5um thickness) were prepared and stained with haematoxylin and eosin (Hx\& E), Masson's trichrome stain and periodic acid - Schiff technique. ${ }^{[16] \text { and }[17]}$

\section{2- Electron microscopic study:}

Semithin sections were cut and stained with toluidine blue for studying the structure of the cardiac muscle fibres .The chosen regions were cut with glass knives for ultrathin sections (60-70 nm) which mounted on copper grids.${ }^{[18]}$ The ultra- thin sections were post stained with $2 \%$ uranyl acetate and lead citrate and examined with Joel TEM CS 100 electron microscope.

3- laboratory tests:

Venous blood samples were withdrawn from the retro-orbital plexus in heparinized capillary tubes, by pressing the thumb behind the angle of the jaw resulting in the engorgement of the retro-orbital plexus . ${ }^{[19]}$

After separation of plasma from blood, the various biochemical parameters were estimated as the following.

a- Total serum cholesterol (TC) . ${ }^{[20]}$

b- Triglycerides (TG). ${ }^{[21]}$

c- Low density lipoprotein cholesterol, $($ LDL $)($ asLDL $-\mathrm{C}=$ Total cholesterol $(\mathrm{TC})-$ (HDL-C+ VLDL-C). ${ }^{[22]}$

d- High density lipoprotein cholesterol, (HDL). ${ }^{[23]}$

e- Very low density lipoprotein (vLDL)(as VLDL-C $=$ Triglycerides / 5). ${ }^{[22]}$

f- Atherogenic index(AI) (as Atherogenic Index $(\mathrm{AI})=\mathrm{TG} / \mathrm{HDL}-\mathrm{C}) .^{[22]}$

\section{4- statistically evaluation:}

The statistical data included were expressed as mean, standard deviation( $\mathrm{SD} \pm$ ) and the student $\mathrm{T}$ test was used to elucidate the differences between different groups and the control group ${ }^{\left[{ }^{24]}\right.}$ The obtained data were analyzed by using SPSS (Statistical package for social studies) software (V.16), the level of significance was adopted at $\mathbf{P} \leq \mathbf{0 . 0 5}$.

\section{RESULTS}

I- The histological results:-

1- Control group (C) (Figs. 1-8).

- Light microscope study (LM): branched striatedcardiac muscle fibers were detected in the control group with single centrally located oval nuclei andintercalated discs.

- Normal fine collagen fibres were observed between the cardiac muscle fibres with normal distribution of PAS + ve materials (magenta red) in sarcolemma of the cardiac muscle fibres.

- Semithin sections revealed visible cross striations and central vesicular nuclei surrounded by a clear perinuclear zone.

- Electron Microscope study: (EM) revealed that cardiomyocytes contain strands of myofibrils formed of light bands, $\mathrm{Z}$ lines, dark bands, $\mathrm{H}$ zone and $\mathrm{M}$ line bisected $\mathrm{H}$ zoneand mitochondria with normal organization of cristae.

2- Hyperlipidemic group (H) (Figs. 9-17).

- LM revealed indistinct and distorted striation of fibres, separation of muscle fibres with vesicular nuclei and presence of myofibroblast.

- Mild to moderate increase in collagen fibres with decreased PAS+ ve materials in the cardiac muscle were observedregarded to statistically significance data in comparisonwith the control group as $\mathrm{P}<0.05$

- Semithin sections revealed loss of striation of muscle fibres, congested and dilated blood vessels, loss of some nuclei and presence of myofibroblasts.

- EM revealed an area of loss of striation, appearance of nucleus of myofibroblast ,splitting of muscle fibres with condensation of nuclear chromatin, normalintercalated discs and $\mathrm{Z}$ line with normal mitochondria.

3- Treated group one (T1)(Figs. 18-24).

- LM revealed that cardiac muscle fibers restored their striation with pyknotic nuclei, increased myofibrobasts and appearance of lymphocytic infiltration .

- Mild decrease in collagen fibres with mild increase in PAS + ve materials in cardiac muscle were observed regarded to statistically significant data in comparison with hyperlipidemic group.

- Semithin sections revealed myofibrobast nucleusand lymphocytic infiltration.

- EM revealed nucleus of myofibroblast, congested blood vessels with areas of loss of striation, nuclear damage with separated and 
splitted myofibres, mitochondrial vacuolation and disorganization of their cristae.

4- Treated group two (T2) (Figs. 25-34).

- LM revealedpatchy loss of striation with splitted muscle fibres, some nuclei become pyknotic with margination of nuclear chromatin (Retipersion), congested blood vessels with presence of lymphocytic infiltration.

- Increased collagen fibres was noticed \&focal areas of excessive collagen fibres deposition were also seen with decreased PAS + ve materials in the cardiac muscle were observed regarded to statistically significance data in comparison with hyperlipidemic and treated 1 groups.

- Semithin sections revealed loss of striation, dilated and congested blood vessels with vacuolation and nuclear damage.

- EM revealed indentured degenerative pyknotic nuclei of cardiomyocytes with patchy loss of striation, nucleus of myofibroblast ,swollen mitochondrial with expansion of the inter-membranous spaces and disorganization of their cristae.

5- Recovery group one (R1) (Figs. 35-37).

- LM revealed that cardiac muscle fibres restored their normal striation and myofibroblast.

- Decreased collagen fibres with increased PAS + ve materials in the cardiac muscle were observedregarded to statistically significant data in comparison with hyperlipidemic, treated $1 \& 2$.

6- Recovery group two (R2)(Figs. 38-40).

- LM revealed that cardiac muscle fibres restored their normal striation with decreased collagen fibres with increased PAS + ve materialsin the cardiac muscle were observedregarded to statistically significant data in comparison with hyperlipidemic , treated $1 \& 2$ and recovery 1 groups.

\section{II-Statistically and laboratory evaluated data:-}

1- Table. 1 : showing a significant difference in percentage of collagen fibres between the cardiac muscle fibers in thedifferent groups when compared to the control group specially in $\mathrm{T} 2$ group as $\mathrm{p}<0.05$.

2- Table. 2:showing a significant difference in optical density of PAS + ve materials in the cardiac muscle fibres in the different groups when compared to the control group especially in $\mathrm{T} 2$ group as $\mathrm{p}<0.05$.
3- Tables. $3 \& 4 \& 5 \& 6 \& 7$ and 8: showing significant increase in levels of cholesterol, Triglycerides, Low Density Lipoprotein Cholesterol (LDL-C) very low density lipoprotein (vLDL) and atherogenic index $(\mathrm{AI})$ and significant decrease of High Density Lipoprotein Cholesterol (HDL-C) between the control and hyperlipidemic groups as $\mathrm{p}<0.05$.

Administration of ATOR to treated groups induced significant decreases in the serum levels of cholesterol , Triglycerides, Low Density Lipoprotein Cholesterol (LDL-C) very low density lipoprotein (vLDL) and atherogenic index(AI) and significant increase of High Density Lipoprotein Cholesterol (HDL-C)when compared with hyperlipidemic group as $\mathrm{p}<0.05$.

Also there were significant decreases in the serum levels of cholesterol , Triglycerides, Low Density Lipoprotein Cholesterol (LDLC) very low density lipoprotein (vLDL) and atherogenic index(AI)and significant increase of High Density Lipoprotein Cholesterol (HDL-C) in recovery groups when compared with hyperlipidemic and treated groups $(\mathrm{p}<$ 0.05).

\section{DISCUSSION}

Hyperlipidemia is a heterogeneous disorder commonly characterized by an increased flux of free fatty acids (FFAs), raised cholesterol ,triglycerides (TGs), lowdensity lipoprotein-cholesterol and apolipoprotein B (Apo-B) levels, as well as by a reduced plasma high-density lipoprotein -cholesterol concentration because of metabolic effects or dietary and lifestyle habits . ${ }^{[25]}$

High-fat diet as well as hyperlipidemia might induce oxidative stress and lipid oxidation that contributes to the high risk of cardiovascular diseases (CVD).$^{[26]}$

Atorvastatin (Ator) (HMG-Co-A reductase inhibitor)was shown to be a highly effective member of statinswhich are used to reduce cardiovascular-related morbidity and mortality in patients with or without coronary artery disease . ${ }^{[27]}$

Indications for statins therapy as recommended by The National Institute for Health and Clinical Excellence make statins one of the world's most prescribed drugs. One of these indication was hypercholesterolemia.$^{[28]}$ 
Reducing the level of cholesterol in blood plasma is an effective method to treat hyperlipidemia-related diseases. The currently used medicines, such as fibrates and statins, are characterized by their high lipidlowering speed and efficacy. ${ }^{[29]}$

Myotoxic effects of atorvastatin are known to be dose and time dependent. As pointed out, impaired metabolism of statins, pharmacokinetic interactions and or genetic effects are all probable causes of the myotoxicity. ${ }^{[30]}$

Little studies are available about Ator effect on structure of the cardiac muscle fibres.The present study tried to evaluate the adverse effects of Ator on cardiac muscle fibres in rats, at the first induction of hyperlipidemia by high fat diet for 3 weeks , following by use of Ator in dose equivalent to human dose for 3 and 6 weeks ,then the treatment was stopped for 3 and 6 weeks with using normal diet, to explain if these adverse effects are reversible or not, the main parameters of this study depend on light and electron microscopy examination of the cardiac muscle fibres.

The obtained results in the present study revealed that the main histological changes in hyperlipidemic group $(\mathbf{H})$ at level of LM, showedindistinct and distorted striation in the cardiac muscle fibres with separation of muscle fibres, vesicular nuclei, loss of some nuclei, congested and dilated blood vessels and presence of myofibroblast . But at level of EM there were condensation of nuclear chromatin with normal mitochondria.

These results are in agreements with Haddad et al. ${ }^{[31]}$ who reported that the heart showed some sorts of haemorrhages and wavy appearance of myofibres, as well as the sarcoplasm of some fibres was characterized by granular appearance, large areas of degenerated muscle fibers with evident loss of transverse striations and wide interfascicular spaces in hyperlipidemic group compared to the control group.

Ekhteraeet al $^{[32]}$,reported that there were a close relationship between muscle degeneration and ultrastructural abnormalities in mitochondria although there was a discrepancy concerning the time of earliest mitochondrial change.

Evans and Rees ${ }^{[33]}$, reported that statinassociated myotoxicity is complex, involving effects on cell membrane structure and function, mitochondrial dysfunction and impaired myocyte regeneration.

In thisstudy the main histological changes in Ator treated group one (T1) at level of LMinvolvingrestoration of the cardiac muscles striation and pyknotic nuclei, increased myofibrobasts and lymphocytic infiltration. But al level of EM there were congested blood vessels with areas of loss of striation and nuclear damage with separated and splitted myofibres, mitochondrial vacuolation and disorganization of theircristae.

In thisstudy the main histological changes in Ator treated group two (T2) at level of LM, involvingpatchy loss of striation with splitted muscle fibres, some nuclei became pykinotic with margination of nuclear chromatin (Retipersion), congested blood vessels and presence of lymphocytic infiltration. But al level of EM there were many degenerated and pyknotic nuclei of cardiomyocytes, nucleus of myofibroblast and mitochondrial destructive swelling disorganization of cristae.

The obtained results in this study are in arrangement withthe work ofJosephet al. ${ }^{[34]}$, who observed ultrastructural changes in the cardiac muscle fibres after long-term Ator treatment .They reported that treated hearts presented scattered, misorganized and swollen mitochondria, protein aggregates as well as inclusion bodies and enlarged, mislocalized T-tubular structures.

In correlation with the present work Noory et $\boldsymbol{a l} .{ }^{[35]}$, reported in he'swork that atorvastatin administration in rats led to ultrastructural changes in the cardiac muscle fibres with clear rupture of some muscle fibres with separation of them from each other together with mitochondria swelling and cristae destruction. Discontinuous and elongation of the cardiac muscle fibres were observed which might be due to occurrence of partial decomposition in these muscles.

In thisstudy the main histological changes after stopping treatment with Ator in the treated groups with normal diet, in recovery group one (R1) showed restored striation and myofibroblasts . Recovery group two (R2) showed nearly normal structure of the cardiac muscle fibres.

The above obtained results are in arrangement with those of Catapano ${ }^{[10]}$, who reported that after stopping of atorvastatin 
,side effects disappeared and this usually leading to a full recovery.

In this study appearance of myofibroblast in different groups may be support results in increase of collagen fibres in different percentage, also tougher with lymphocytic infiltration may be indicated heart injury in response either to hyperlipidemia and Ator treatment.

The above obtained results are in arrangement withthose of Jennifer and Heather ${ }^{[36]}$, who reported that in the case of ischemia or heart failure, the myofibroblasts can be found in large areas of the heart as myofibroblasts are not part of normal cardiac tissue and appear only following cardiac injury. Myofibroblasts produce and secrete a number of cytokines themselves which help to maintain the inflammatory response to injury.

The statically evaluated data in this study declared a significant difference between all groups in collagen fibres percentage especially in treated group two as it shown a marked increase of collagen fibres. Also the optical density of PAS + materialsshowed a significant difference between all groups especially in treated group two as it shown a marked decrease in PAS + materials.

In this study successfully induced hyperlipidemia in rats modulating the biochemical parameters of lipid profile, enhancement levels of cholesterol, triglycerides, low density lipoprotein cholesterol (LDL-C) very low density lipoprotein (vLDL) and atherogenic index(AI) were elevated and high density lipoprotein cholesterol (HDL-C) was decreased. These results consistent with Farhanaet al. ${ }^{[37]}$, who reported that induction of hyperlipidemia resulted in several alterations in serum cholesterol, triglycerides, LDL-c, VLDL, atherogenic index and HDL-c levels.

The hypercholesterolemic effect induced by HFD may be due to the activity of the ratedetermining enzyme in cholesterol biosynthesis, HMG-CoA reductase, stimulating the cholesterogenesis rate ${ }^{[38]}$. Liu et al. [39], has suggested that increased lipogenesis in cholesterol-fed rats due to decreased oxidation of fatty acids and decreased secretion of VLDL as causes for the accumulation TG and decrease lipolysis of fat cells. Yokogoshi et al . ${ }^{[40]}$, thought that increased cholesterol synthesis in the high-cholesterol-fed rats. Xuet al. ${ }^{[41]}$, has also reported that the impaired hepatic lipid homeostasis because of lipid accumulation attributed to the increasing activity of the enzymes involved in fatty acid biosynthesis in the rats by the dietary cholesterol.

In the present study administration of Ator to the treated groups induced significant decreases in the levels of cholesterol, triglycerides, low density lipoprotein cholesterol (LDL-C) very low density lipoprotein (vLDL) and atherogenic index(AI) with a significant increase of high density lipoprotein cholesterol (HDL-C), these result are in accordance with those of Bethet al . [42], who reported that administration of Ator to rats fed HFD caused a reduction in serum TC and LDLc levels, VLDLlevel with a consequence reduction in atherogenic index, while serum HDL-c levels were elevated.

These findings could be interpreted by the inhibitory effect of Ator on HMG-CoA reductase enzyme which catalyzes the conversion of HMG-CoA to mevalonate, a rate-limiting step in the formation of endogenous cholesterol leading to the decrease in the intracellular stores of cholesterol.This in turn results in the upregulation of LDL receptors on the cell membrane, thus increasing the clearance of LDL-c from plasma. ${ }^{[43]}$ Another possible explanation of our results is that Ator may lower LDL-c level by inhibiting hepatic cholesterol synthesis in very low density lipoprotein (VLDL) which is the source of LDL-c. Thus may impair VLDL particle assembly and secretion from liver, decrease the VLDL levels in plasma, and further decrease the LDL level in plasma. ${ }^{[4]}$

Other proposed mechanism for Ator in lowering serum cholesterol level is by inhibiting the absorption of dietary cholesterol. Statins may reduce the cholesterol content in the intestinal mucosal cells, with a subsequent reduction in acyl-CoA cholesterylacyltransferase (ACAT) activity, which catalyzes the intracellular esterification of cholesterol and formation of cholesteryl esters, and thus decrease the absorption of cholesterol. ${ }^{[45]}$

The reduction in HDL cholesterol level in animals fed high fat diet may be due to the 
decrease in lecithin-cholesterol acyltransferase (LCAT) activity, the enzyme involved in the transesterification of cholesterol, the maturation of HDL and the flux of cholesterol from cell membranes into HDL. ${ }^{[46]}$ Atorvastatin(Ator) treatment could restore HDL-c levels may be attributed to the reduction of cholesteryl ester transfer protein (CETP) which plays an important role in HDL metabolism. [47] Atorvastatin(Ator appeared to reduce triglyceride levels by increasing LDL clearance and by inhibiting triglyceride synthesis. It also increases HDL cholesterol levels . ${ }^{[48]}$

Conclusion:this study shed light on Ator side effects on cardiac muscle fibres, so it should not stop on its role in modulating lipid profile . So patients on Ator treatment need to adjust time and dose or try to use another method to modulate lipid profile as change diet habits or use another safe medication as fibrates and herbal medicinal plants. Also advice for another researches on Ator adverse effect and comparison between the different kinds of statins to select themost safest one on the cardiac muscle fibres.

\section{REFERENCES}

1- Law MR (1999): Lowering heart disease risk with cholesterol reduction: evidence from observational studies and clinical trials. Eur. Heart J. Suppl. ,1:3-8.

2- Karen Sliwa and Ana Mocumbi (2009): Forgotten cardiovascular diseases in Africa. Clin. Res .Cardiol., 10:1007-1011.

3- Doha AM ,Thanaa EH andSahar YA (2010): Reduction in hypercholesterolemia and risk of cardiovasculardiseases by mixtures of plant foodextracts: a study on plasma lipid profile, oxidative stress and testosterone in rats. Grasas Y aceites., 61(4): 378-389.

4- Deshmukh HA, Colhoun HM, Johnson T, McKeigue PM, Betteridge DJ, Durrington PN, Fuller JH, Livingstone S, Hitman GA (2012):"Genome-wide association study of genetic determinants of LDL-c response to atorvastatin therapy: importance of Lp(a)". J Lipid Res ., 53 (5): 1000-1011.

5- Molokhia M, McKeigue P, Curcin V andMajeed A ( 2008): Statin induced myopathy and myalgia: time trend analysis and comparison of risk associated with statin class from 19912006. PLoS One., 3:e2522.

6- Pfizer (2008): Annual Report.

7- Kobayashi M, Chisaki I and Narumi K (2008):. Association between risk of myopathy and cholesterol-lowering effect: a comparison of all statins. Life Sci., 82: 969-975.
8- Ahmida, MH andAbuzogaya MH (2009) : The effects of oral administration of green tea and ginger extracts on serum and hepatic lipid content in rats fed a hyperlipidemic diet. Der. Pharmacia. Lettre., 1 (1): 56-64.

9- Armitage J (2007) : The safety of statins in clinical practice. Lancet.,370:1781-1790.

10- Catapano AL. (2012): Statin-induced myotoxicity: pharmacokinetic differences among statins and the risk of rhabdomyolysis, with particular reference to pitavastatin. CurrVascPharmacol., 10(2):257-67.

11- Abdou HMandEIMazoudy RH (2011) :Oxidative damage, hyperlipidemia and histological alterations of cardiac and skeletal muscles induced by different doses of diazinon in female rats.J Hazard Mater., 15;182(1-3):273-278.

12- Shyamala MP, Venukumar MR andLatha MS (2003): Antioxidant potential of the Syzygiumaromaticum (Gaertn) Linn (Cloves) in rats fed with high fat diet. Indian Journal of pharmacology., 35: 99-103.

13- Schmechel A, Grimm M, El Armouche A, Höppner G, Schwoerer AP, Ehmke H and Eschenhagen T. (2009): Treatment with atorvastatin partially protects the rat heart from harmful catecholamine effects. Cardiovasc.Res., 82(1):100-106.

14- Paget GE , Barnes JM (1964): Evaluation of drug activities, In: Pharmacometrics. Lawrence DR and Bacharach AL, editors, New York :Academic press., 1: 161.

15- Pereira Sand Tettamanti M(2005) :Ahimsa and alternatives - the concept of the 4th R. The CPCSEA in IndiaAltex ., 22 (1): 3-6.

16- Bancroft, JD and Gamble $\mathbf{M ( 2 0 0 2 )}$ : Theory and practice of histological techniques.5th. Ed. Edinburgh. Churchill Livingstone Pub., P: 172175 and P:593- 620.

17- Kiernan JA (1999): Histological and Histochemical Methods Theory and practice $\left(3^{\text {rd }}\right.$ ed).Arnold, A member of the hodder .Headline Group. London .New York and New Delhi.

18- Hayat MA(1986): Basic techniques for transmission electron microscopy. Academic Press Inc.

19- Ghosh MN (2005): Fundamentals of experimental Pharmacology. Edn 3,Hilton and company, Calcutta ., 191-201.

20- Henry RJ, Cannon DC andWinkelman JW (1974): Clinical Chemistry Principles and Techniques, Harper and Row. New York ., 14401452.

21- Fossati $P$ andPrencie $L$ (1982): Serum triglycerides determined colorimeterically with an enzyme that produces hydrogen peroxide. Clin. Chem., 28: 2077-2090.

22- Friedewald, WT, Levy RI andFredrichsor DS (1972): Estimation of the concentration of lowdensity lipioproteincholesterol in plasma without 
use of the preparative ultracentrifuge. Clin. Chem., 18:499-502.

23- Burstein M (1970): Rapid method for isolation of lipoproteins from human serum by precipitation with poly-anion. J. of Lipid Research ., 11: 583 -588 .

24- SnedecorGW, Cochran WG (1980): Statistical Method .United State University Press, single edition, Lowa, London ., P : 59-60.

25- Kolovou GD, Anagnostopoulou KK and Cokkinos DV (2005): Pathophysiology of dyslipidemia in the metabolic syndrome. Postgrad Med J ., 81:358 -366.

26- Holvoet P, Jenny NS, Schreiner PJ, Tracy RP and Jacobs DR(2007): The relationship between oxidized LDL and other cardiovascular risk factors and subclinical CVD in different ethnic groups:the Multi-Ethnic Study of Atherosclerosis (MESA).Atherosclerosis., 94:245 e 52.

27- Jasinska M, Owczarek J and OrszulakMD(2007): Statins: a new insight into their mechanisms of action and consequent pleiotropic effects. PharmacolRep ., 59:483-499.

28- Buettner C, Davis RB, Leveille SG, Mittleman MA and Mukamal KJ (2008): Prevalence of musculoskeletal pain and statin use. J. Gen. Int. Med., 23:1182-1186.

29- Eliasson B, Svensson AM, Miftaraj M, Jonasson JM, Eeg-Olofsson K, Sundell KA andGudbjörnsdóttir S (2011) : . Clinical use and effectiveness of lipid lowering therapies in diabetes mellitus: an observational study from the Swedish National Diabetes Register. PLoSOne., 6: 187-199.

30- Laaksonen R (2006): On the mechanisms of statin-induced myopathy. Clin. Pharmacol. Ther., 79(6):529-531.

31- Haddad AE, Madeha NA and Hanan MA (2013): Efficiency of Barley Bran and Oat Bran in Ameliorating Blood Lipid Profile and the Adverse Histological Changes in Hypercholesterolemic Male Rats. J of BioMed Research International V., 1:1155-1166.

32- Ekhterae D, Lin Z, Lundberg $M S$ and Nunez G(1999) : ARC inhibits cytochrome c release from mitochondria and protects against hypoxiainduced apoptosis in heart-derived $\mathrm{H} 9 \mathrm{c} 2$ cells., Circ Res., 85: 70-77.

33- Evans $M$ and Rees A (2002): Effects of HMGCoA reductase inhibitors On skeletal muscle: are all Statins the same? Drug Saf.,25(9):649-63.

34- Joseph CG, Jan MS, Anna S, Elizabeth KA, Erika AA, Nancy D and Dalton H(2013): Longterm atorvastatin treatment alters cardiac ultrastructure in healthy mice while preserving systolic cardiac function The FASEB Journal ., 27:652-662.

35- Noory TT, Saud AA and Amin A Al-Doaiss (2014): Histological, Histochemical and Ultrastructural Alterations in Male Albino Rats
Treated with Therapeutic Doses of Atorvastatin (Lipitor) and Rosuvastatin (Crestor) Drugs. Kingdom of Saudi Arabia King Saud University College of Science Zoology Department. From PHD on line Abstract.

36- Jennifer Baum and Heather Duffy(2011): Fibroblasts and Myofibroblasts: What are we talking about? J Cardiovasc Pharmacol.,57(4): 376-379.

37- Farhana AD, Sadiq M and Adnan J(2011): Anti -hyperlipidemic effect of acacia honey (DesiKikar) in cholesterol-diet induced hyperlipidemia in rats. Biomedica. V., 27 ;P: $62-$ 67.

38- Bradley-Hillgartner, F., Salati L.M. and Goodridge G (1995): Physiological and molecular mechanisms involved in nutritional regulation of fatty acid synthesis. Physiol. Rev., 75:47-76.

39- Liu CH, Huang $M$, and Huang PC(1995): Sources of triacylglycerol accumulation in livers of rats fed a cholesterol-supplemented diet. Lipids., 30(6):527-531.

40- Yokogoshi H, Mochizuki H, Nanami K, Hida Y, Miyachi F and OdaH(1999):Dietary taurine enhances cholesterol degradation and reduces serum and liver cholesterol concentrations in rats fed a high-cholesterol diet. J Nutr ., 129:17051712.

41- Xu G, Pan LX, Li H, Shang Q, Honda A, Shefer S, Bollineni J, Matsuzaki Y, Tint GS and Salen G (2004): Dietary cholesterol stimulates 15921680373CYP7A1 in rats because farnesoid $\times$ receptor is not activated. Am J PhysiolGastrointest Liver Physiol.,286:730-735.

42- Beth A , Parker PD, Jeffrey A, Capizzi MS, Adam S, Grimaldi BS and Priscilla M (2013): Effect of Statins on Skeletal Muscle FunctionClinical Trial .Circulation .,127:96-103.

43- Blum, CBB ( 1994): Comparison of properities of four inhibitors of 3-hydroxy-3-methy glutaryl coenzyme A reductase. Am. J. Cardiol., 73(14):3D-11D.

44- Bakker-Arkema, RG, Best, J, Fayyad R, Heinonen TM, Marais AD, Nawrocki JW and Black DM (1997): A brief review paper of the efficacy and safety of atorvastatin in early clinical trials. Atherosclerosis., 131(1):17-23.

45- Wang CY, Liu PY and Liao JK. (2008): Pleiotropic effects of statin therapy: Molecular mechanisms and clinical results. Trends Mol.Med.,14(1):37-44.

46- Shepherd J( 1994): Lipoprotein metabolism: an over view. Drugs ., 47:1-10.

47- Guerin M, Egger P, Soudant C, Le Goff W, Dupuis R, and Chapman M J (2002): Cholesteryl ester flux from HDL to VLDL-1 is preferentially enhanced in type IIB hyperlipidemia in the postprandial state., J. Lipid Res. 43: 1652-1660.

48- Ray SK and Rege NN( 2000): Atorvastatin in the management of hyperlipidemia. Journal of post graduate medicine., 46(3): 242-243. 


\section{The Histological Results:}

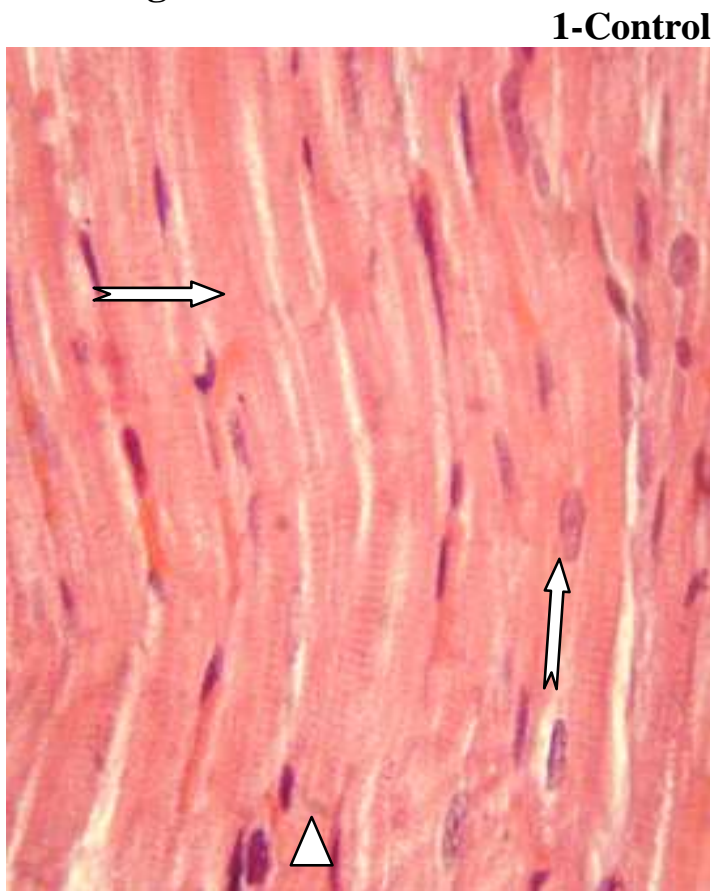

Fig .1: A photomicrograph of cardiac muscle fibres of a rat of group (C) showing striated muscle fibres(transverse arrow) with single centrally located oval nuclei (vertical arrow) and intercalated (arrowhead) (Hx.\& E, X 400).

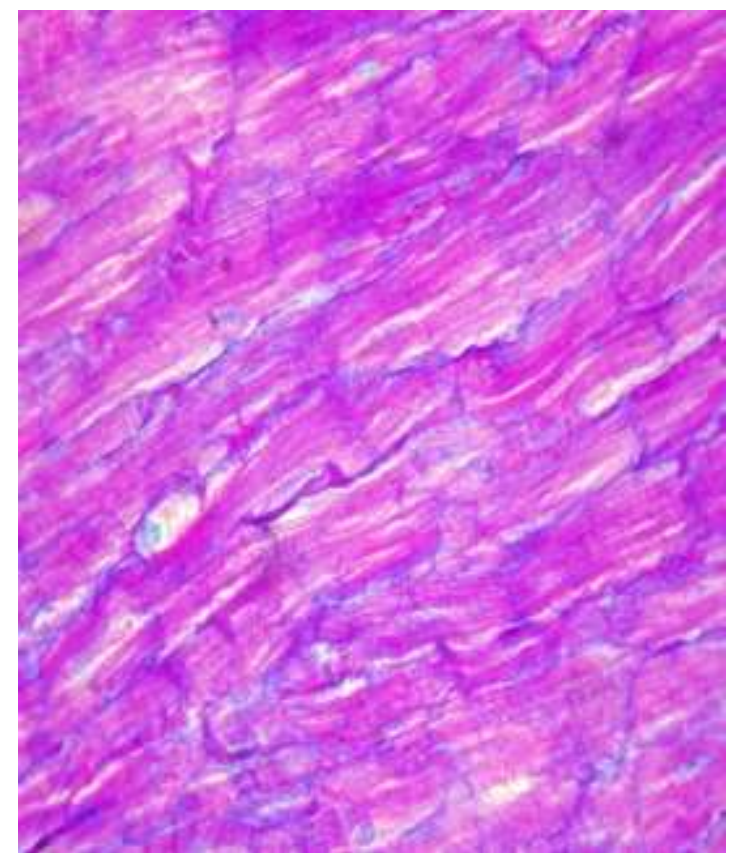

Fig. 3: A photomicrograph of cardiac muscle fibres of a rat of group $(\mathbf{C})$ showing fine collagen fibres between the cardiac muscle fibres (Masson`s trichrome stain $\mathrm{X} 400$ ).

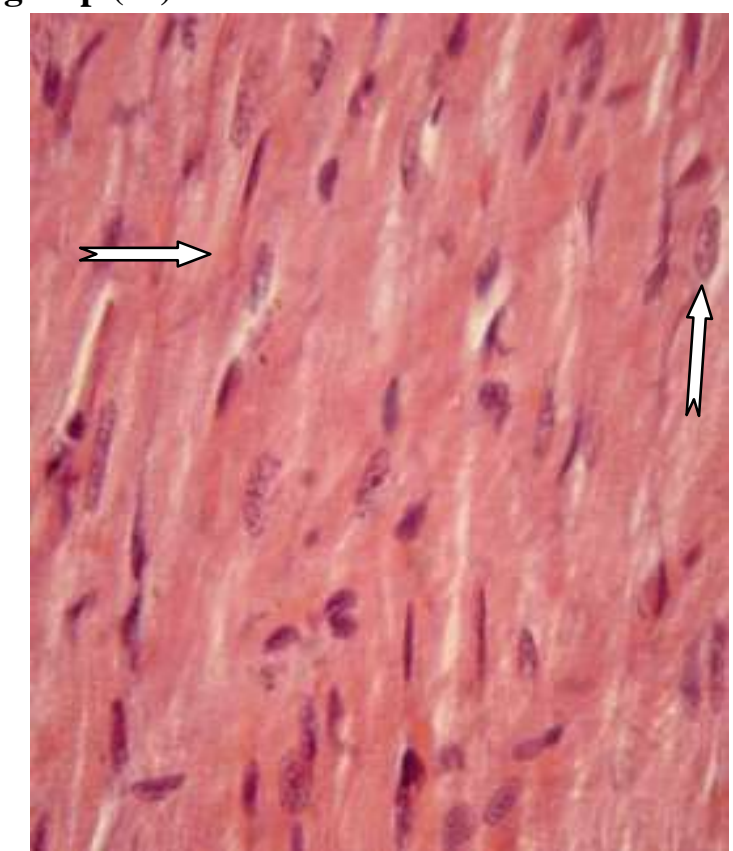

Fig. 2: A photomicrograph of cardiac muscle fibres of a rat of group (C) showing normal branching muscle fibres (transverse arrow) with single centrally located oval nuclei (vertical arrow) (Hx. \& E stain, X 1000).

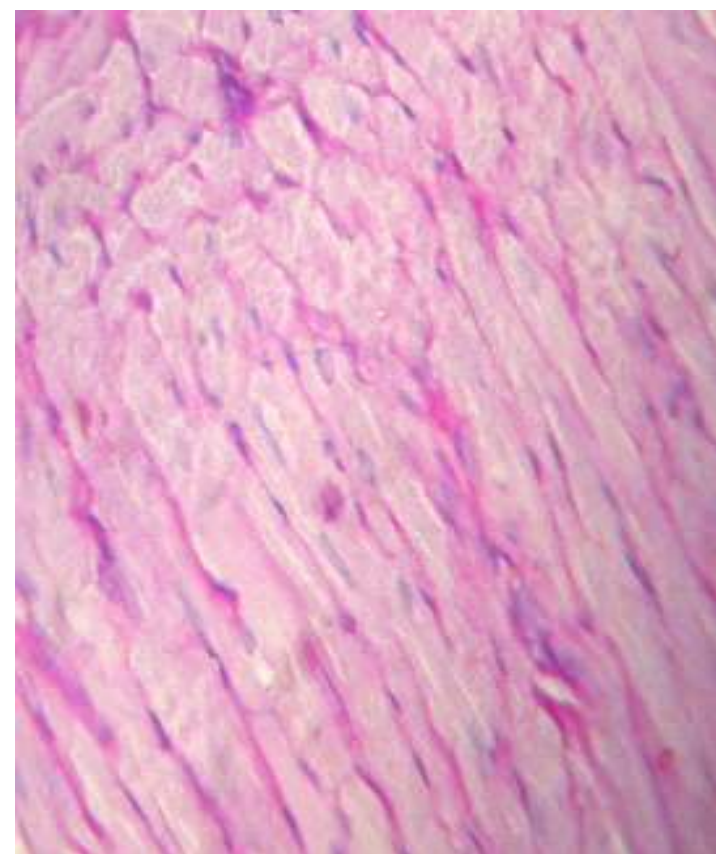

Fig. 4: A photomicrograph of cardiac muscle fibres of a rat of group $(\mathbf{C})$ showing PAS +ve materials(magenta red) in sarcolemma of the cardiac muscle fibres (PAS reaction X 400 ). 


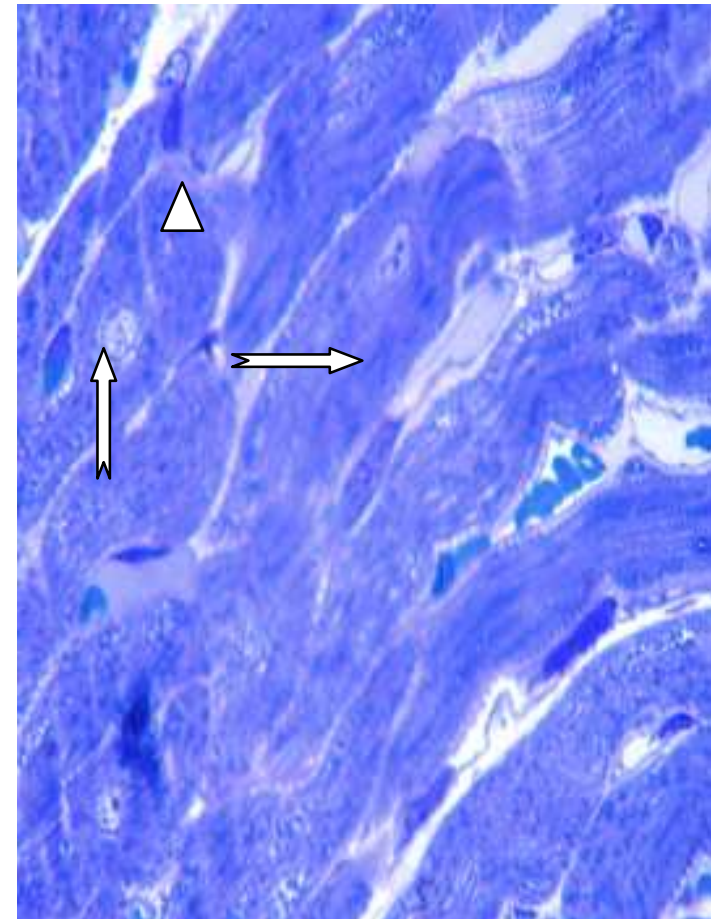

Fig. 5: A photomicrograph of cardiac muscle fibres of a rat of group(C)showing visible cross striations of muscle fibres (transverse arrow) and central vesicular nuclei surrounded by a clear perinuclear zone(vertical arrow) and intercalated, discs (arrowhead) (semithin section ,Toluidine blue stainx1000).

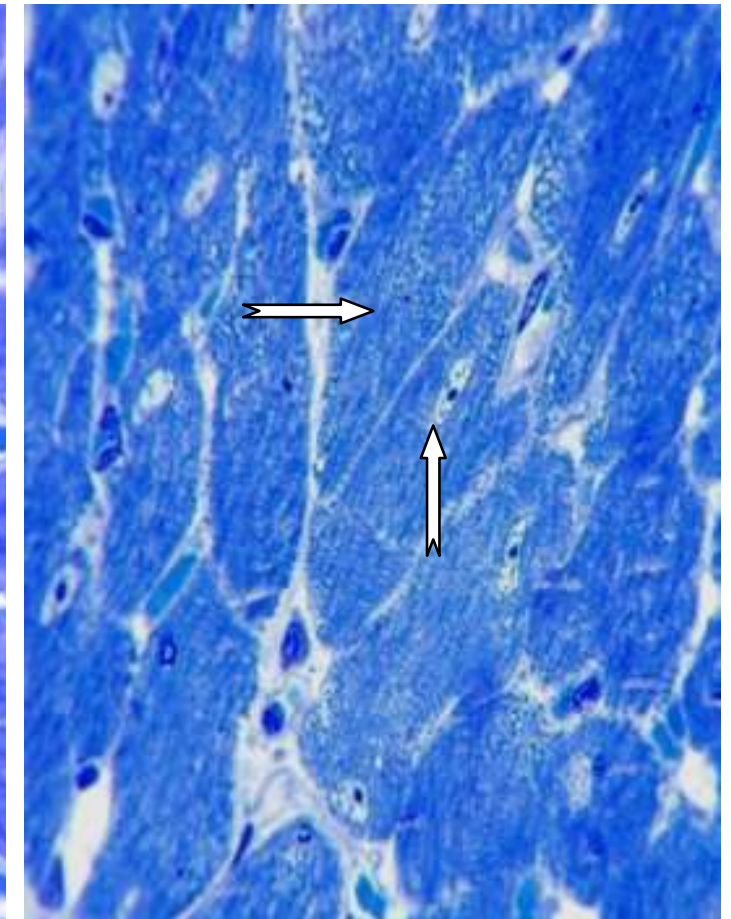

Fig 6: A photomicrograph of cardiac muscle fibres of a rat of group $(\mathbf{C})$ showing fibres with visible striations (transverse arrow)and single central vesicular nuclei (vertical arrow) (semithin section Toluidine blue stain X 1000).

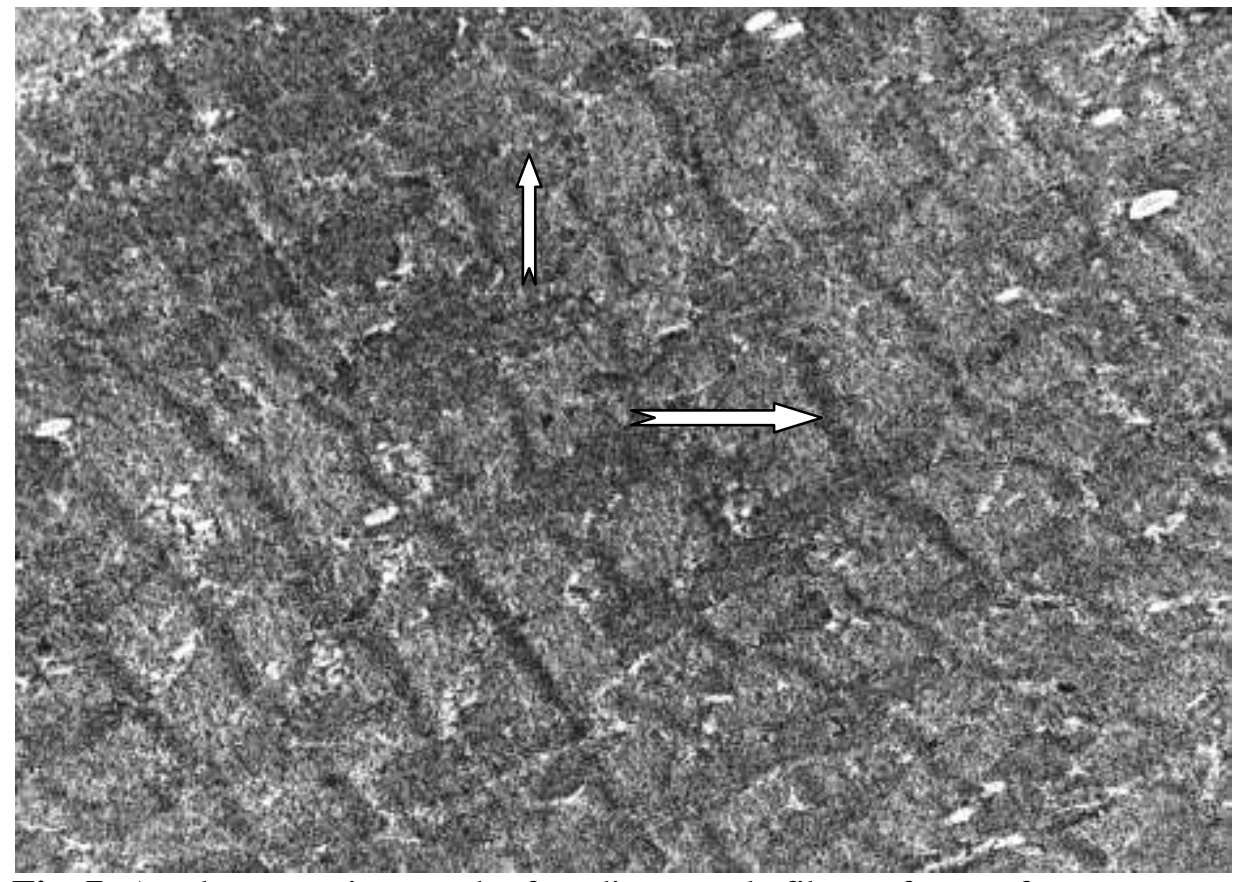

Fig. 7: An electron-micrograph of cardiac muscle fibres of a rat of group $(\mathbf{C})$ showing normal structure of sarcomere(transverse arrow) and striation(vertical arrow) (X10000). 


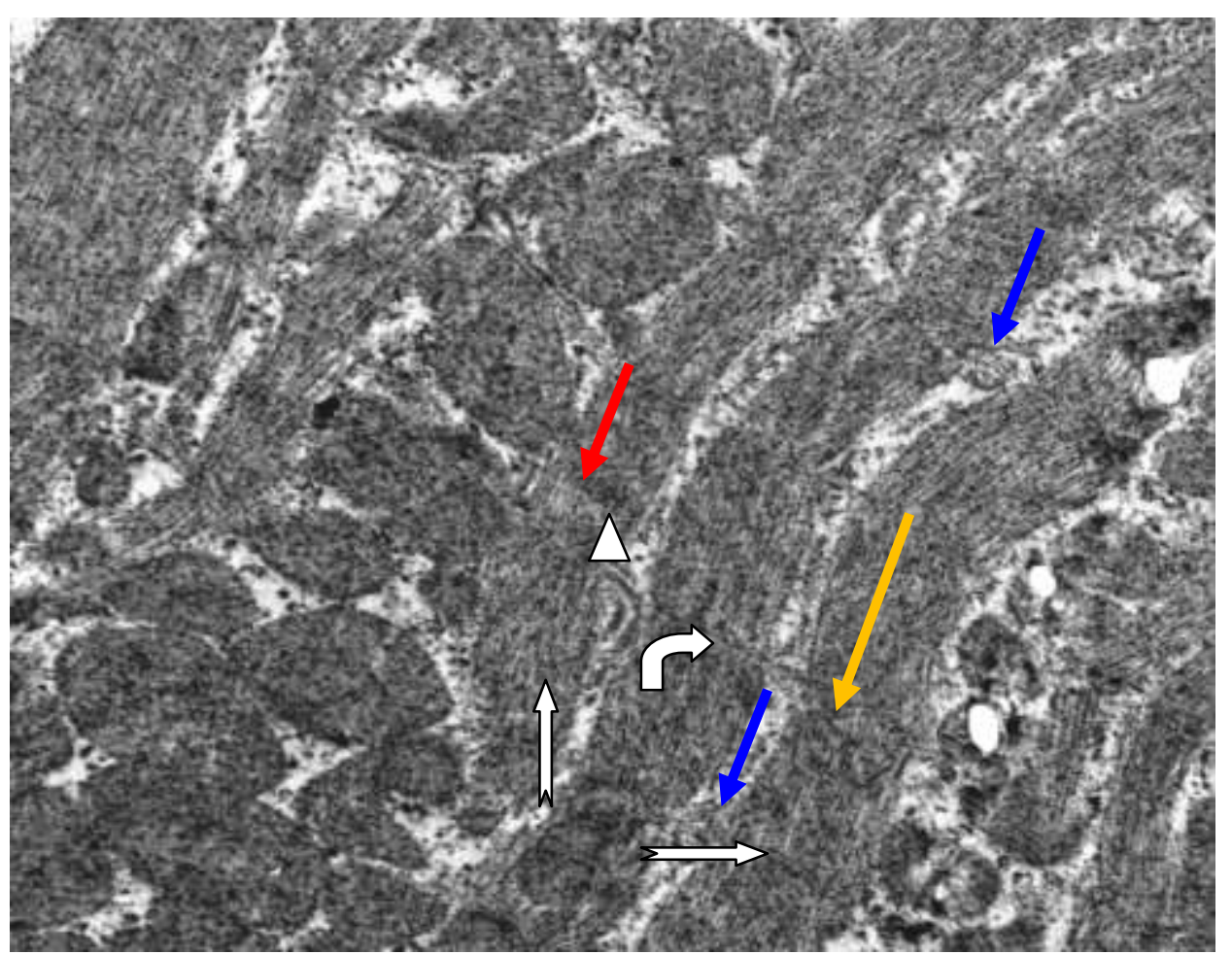

Fig. 8: An electron-micrograph of cardiac muscle fibres of a rat of group $(\mathbf{C})$ showing cardiomyocytes which contain strands of myofibrils formed of light bands (transverse arrow), $\mathrm{Z}$ lines (curved arrow), dark bands(vertical arrow), H zone (arrow head), $\mathrm{M}$ line (red arrow), sarcomere (yellow arrow) and mitochondria with normal organization of their cristae( blue arrow) (X6000).

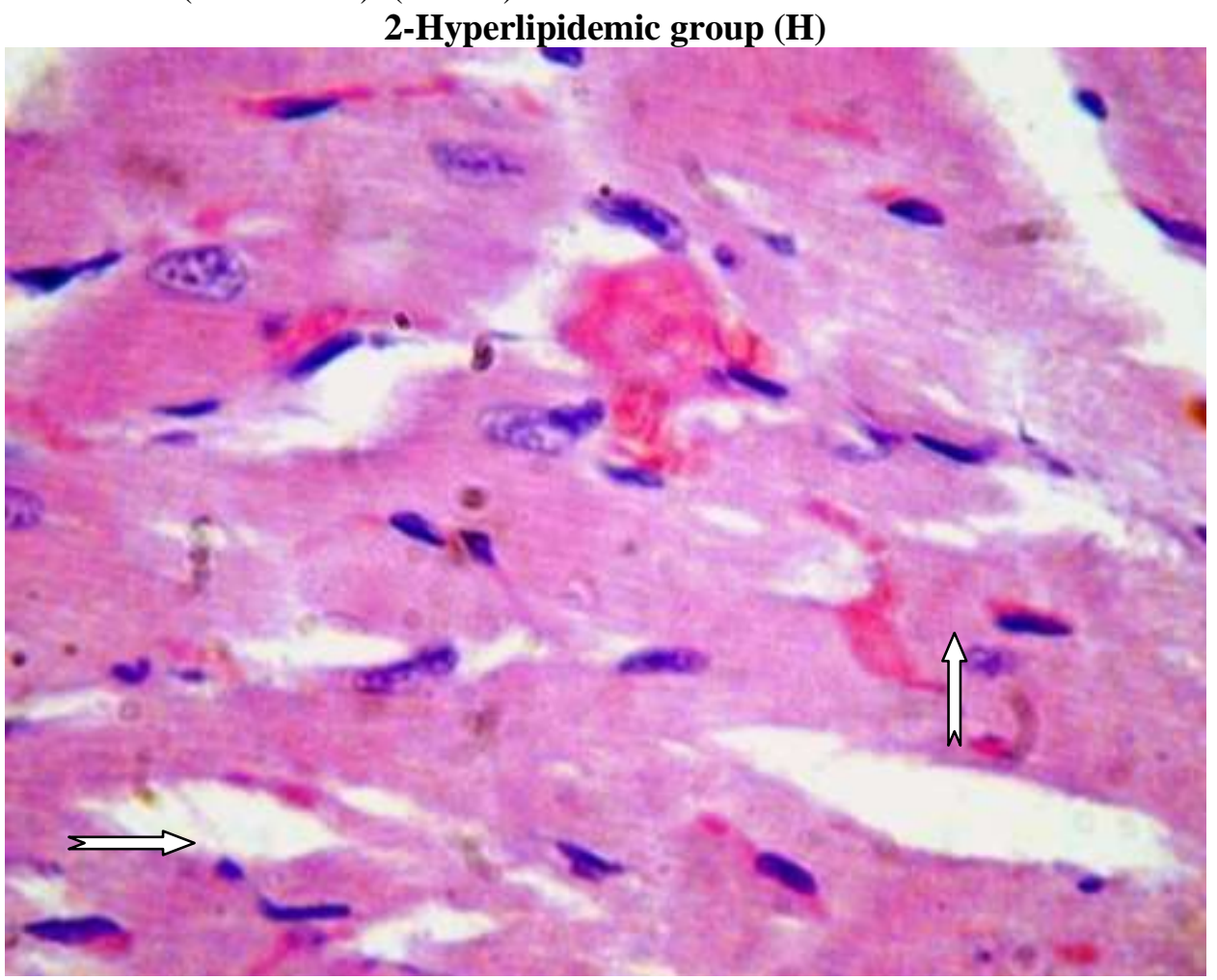

Fig. 9: A photomicrograph of cardiac muscle fibres of a rat of group $(\mathbf{H})$ showing indistinct and distorted striation of muscle fibres(vertical arrow) with separation of them (transverse arrow) ( $\mathrm{Hx} \& \mathrm{E} \times 1000)$. 


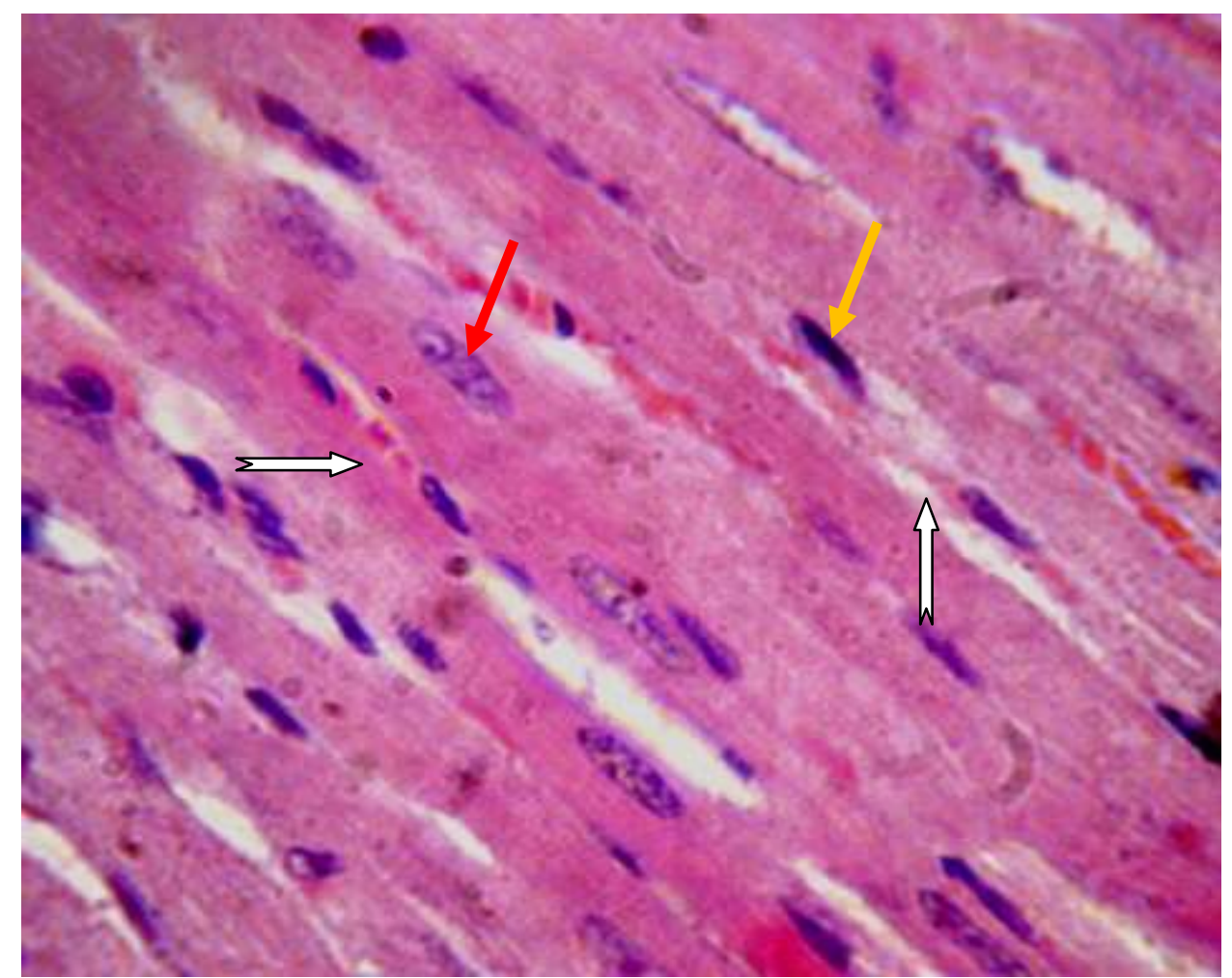

Fig. 10 : A photomicrograph of cardiac muscle fibres of a rat of group $(\mathbf{H})$ showing patches of distorted striation(transverse arrow), separation of muscle fibres (vertical arrow) with vesicular nuclei(red arrow) and presence of myofibroblast (yellow arrow) $(\mathrm{Hx} \& \mathrm{E} \times 1000)$.

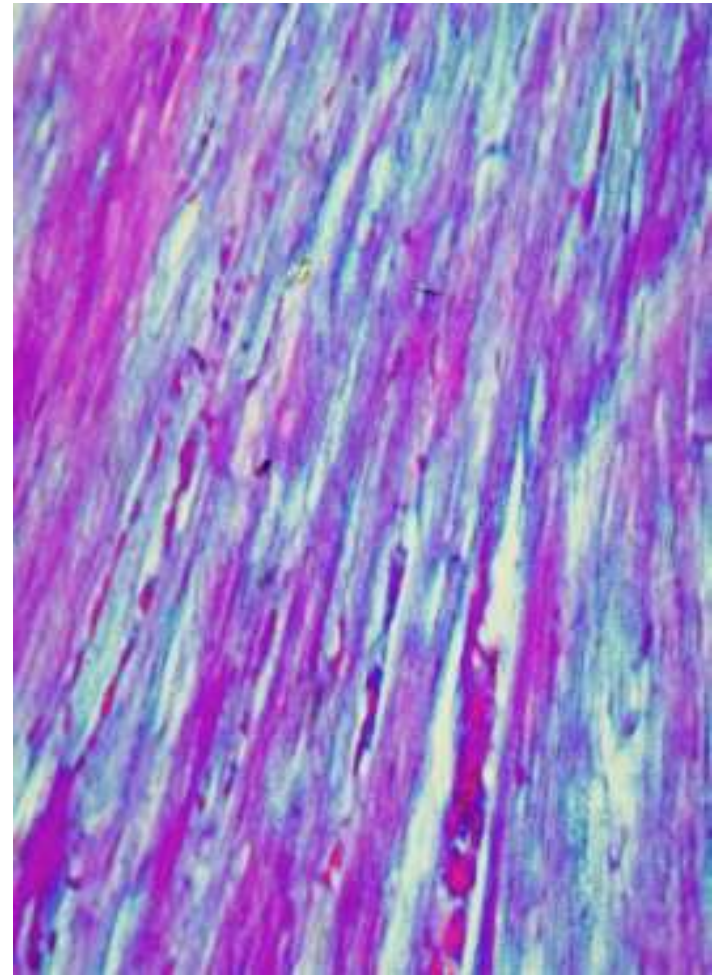

Fig.11:A photomicrograph of cardiac muscle fibres of a rat of group $(\mathbf{H})$ showing mild to moderate increase in collagen fibres between the cardiac muscle fibres (Masson`s trichrome stain X 400 )

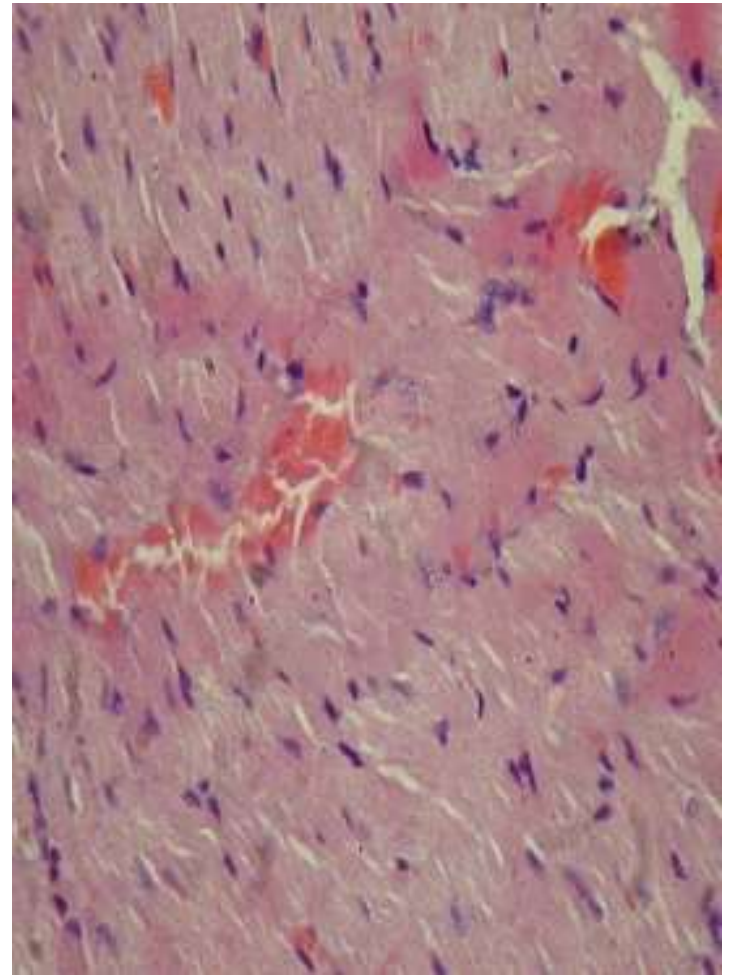

Fig.12: A photomicrograph of cardiac muscle fibres of a rat of group $(\mathbf{H})$ showing decreased PAS+ ve materials in the cardiac muscle fibres .(PAS reaction x 400). 


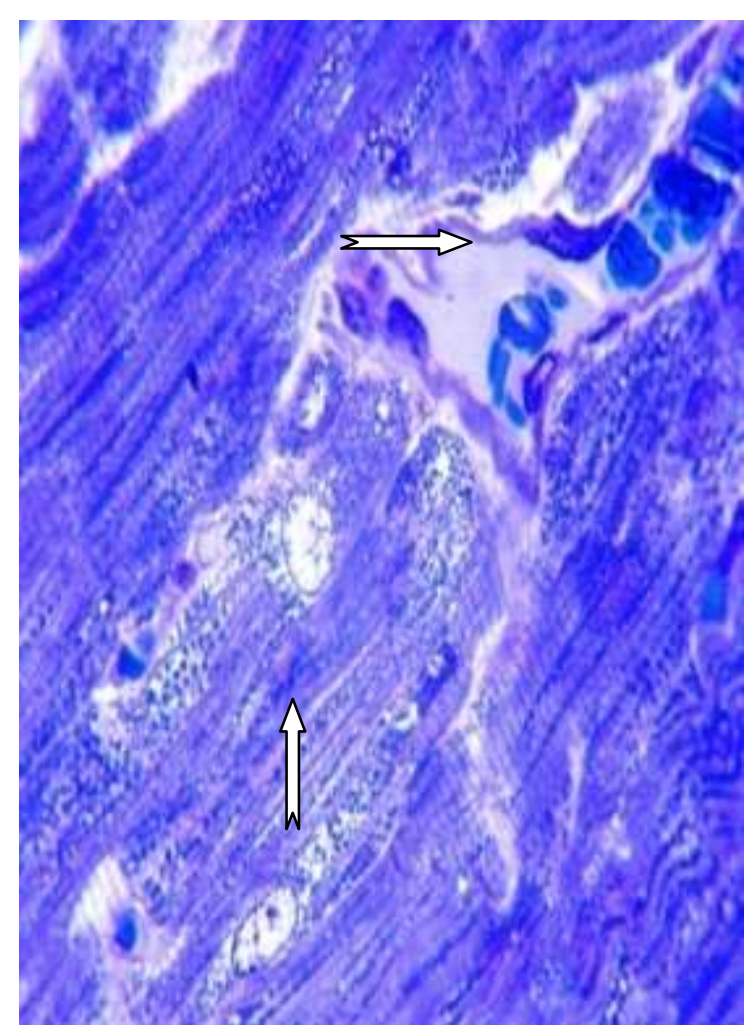

Fig.13:A photomicrograph of cardiac muscle muscle fibres of a rat of group $(\mathbf{H})$ showing distorted striation of muscle fibres (vertical arrow) with congested and dilated blood vessels(transverse arrow) (semithin section ,Toluidine blue stain X 1000).

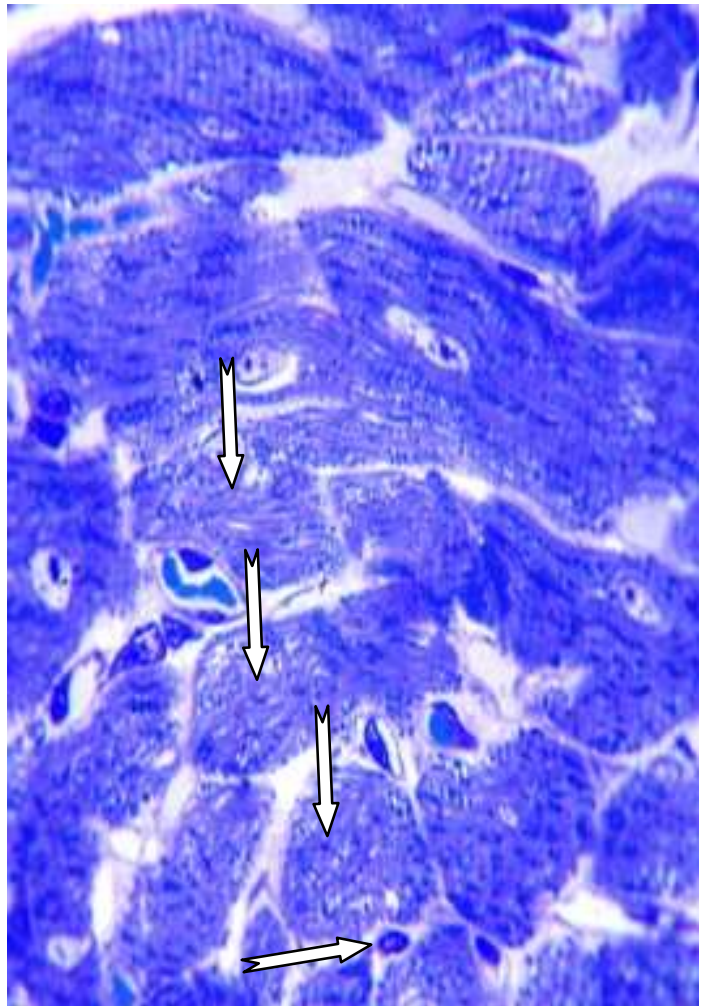

Fig.14: A photomicrograph of cardiac muscle fibres of a rat of group $(\mathbf{H})$ showing cardiac muscle fibres with loss of some nuclei (vertical arrows) and presence of myofibroblasts (transverse arrow) (semithin section Toluidine blue stain X 1000).

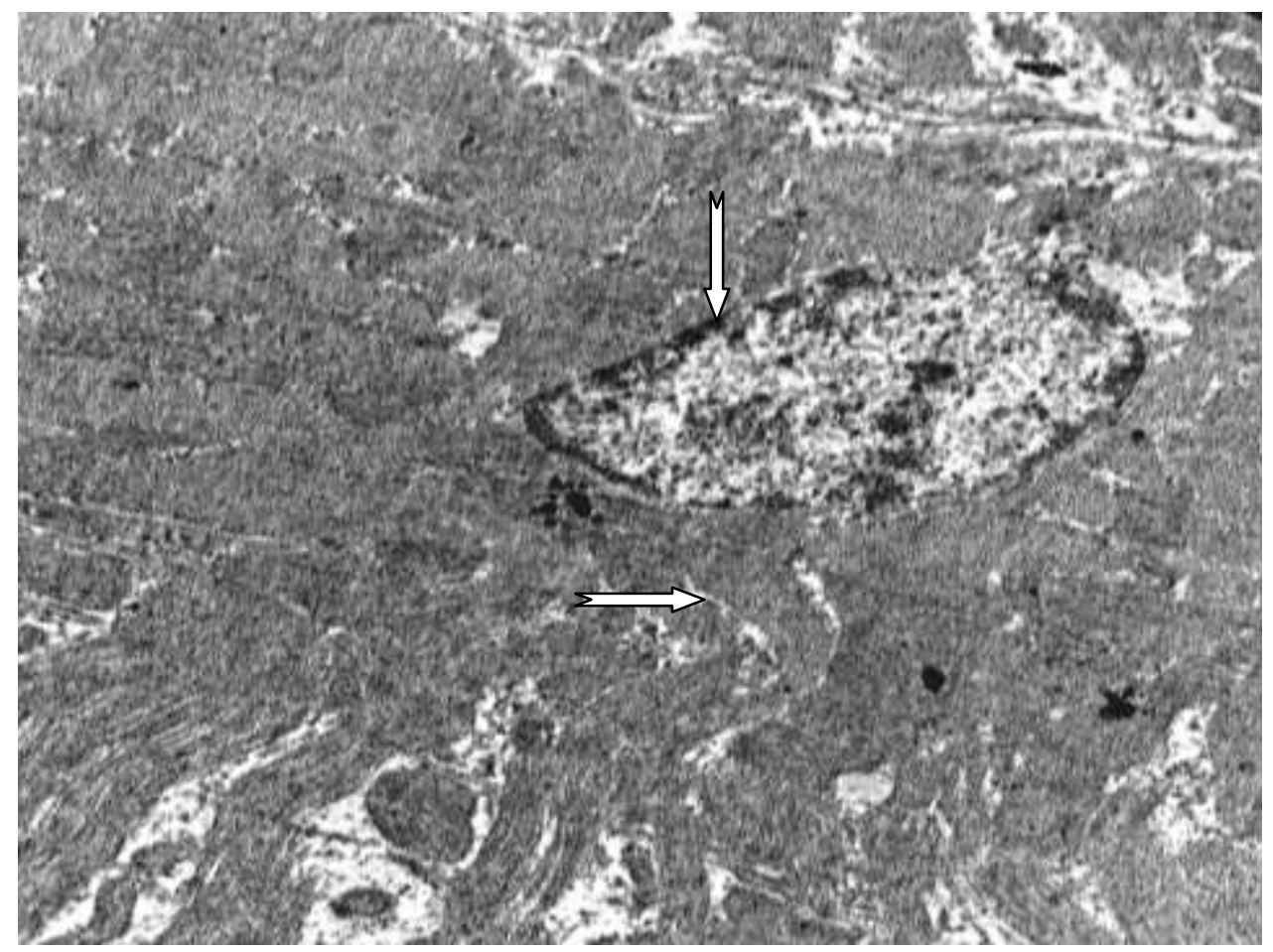

Fig. 15 :An electron-micrograph of cardiac muscle fibres of a rat of group

(H) showing an area of loss of striation (transverse arrow) and vesicular nuclei (vertical arrow) (X12000). 


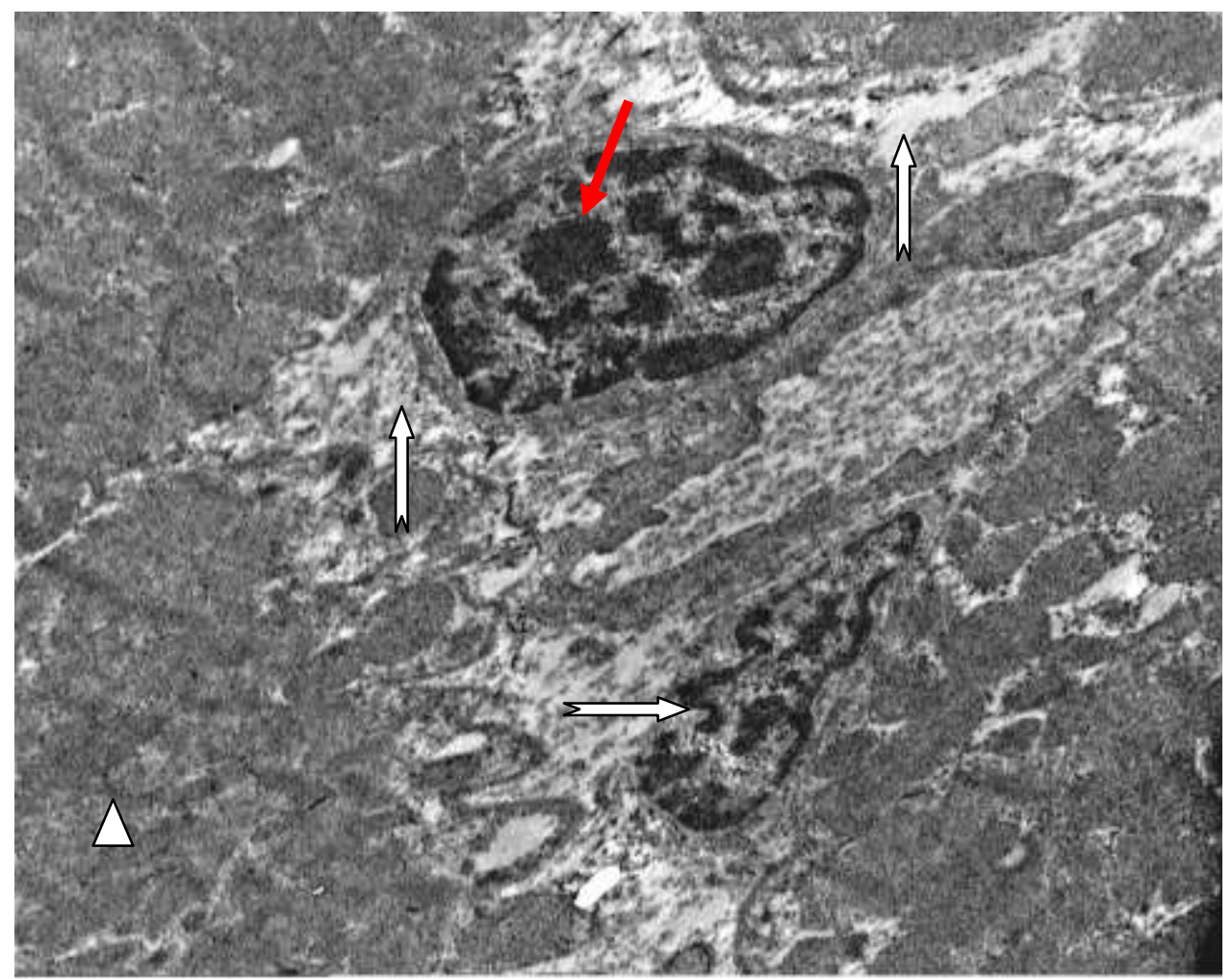

Fig. 16: An electron-micrograph of cardiac muscle fibres of a rat of group (H) showing nucleus of myofibroblast (transverse arrow), splitting of muscle fibres (vertical arrows) with condensation of nuclear chromatin(red arrow) and intercalated disc (arrow head) (X12000).

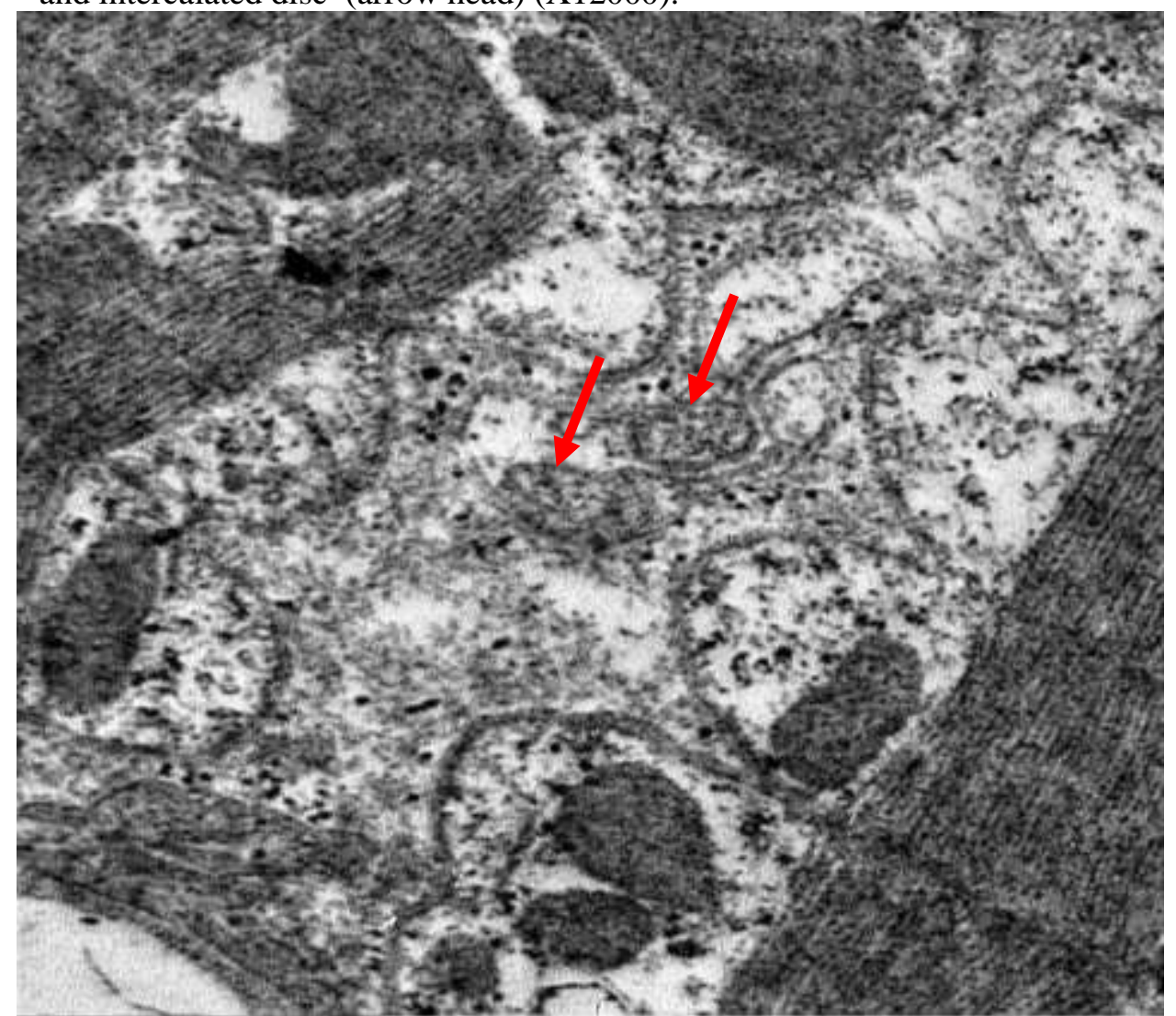

Fig. 17 :An electron-micrograph of cardiac muscle fibres of a rat of group (H) showing some normal mitochondria(red arrows) (X25000). 


\section{3-Treated group one(T1)}

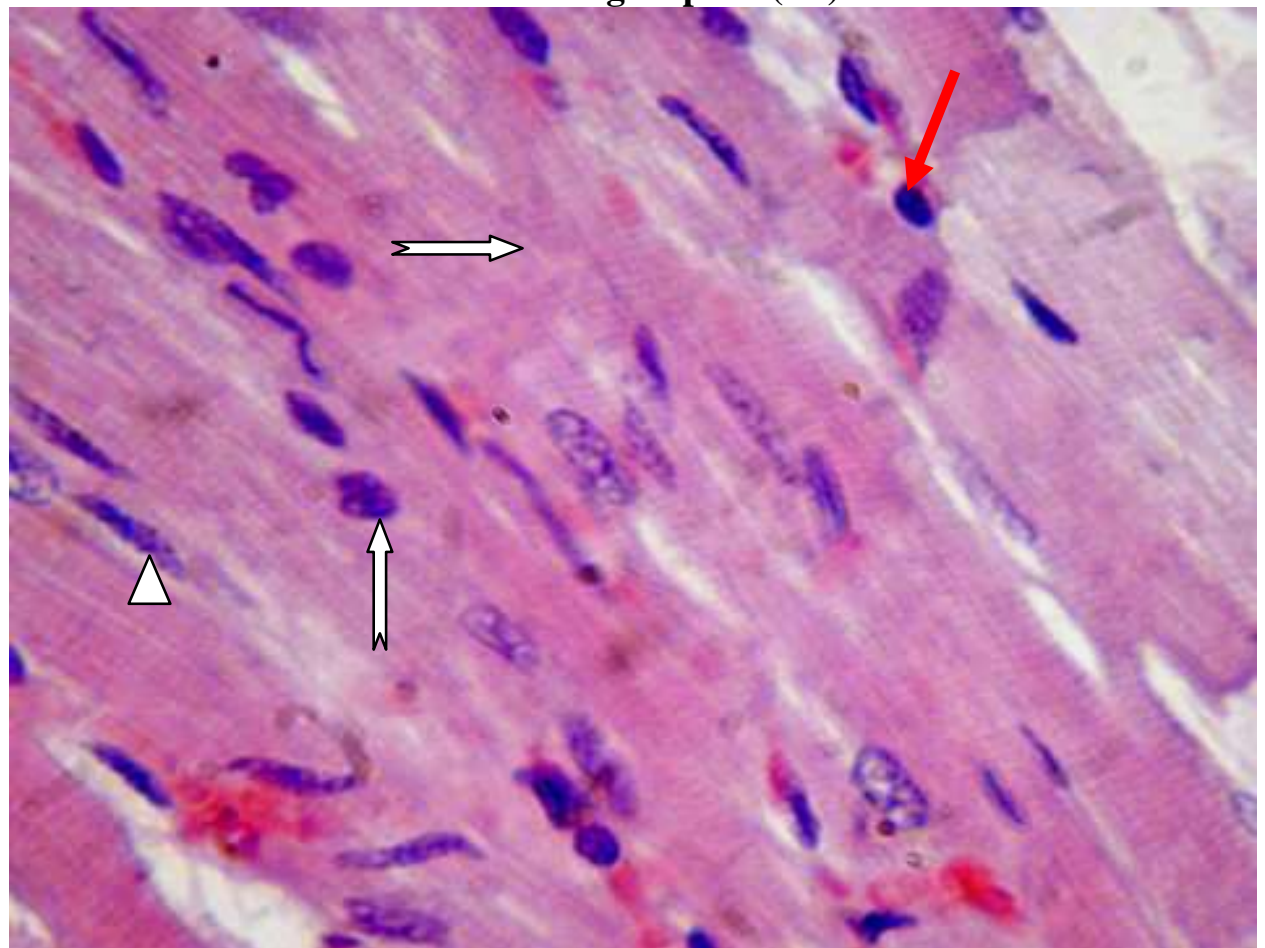

Fig. 18 : A photomicrograph of cardiac muscle fibres of a rat of group (T1) showing cardiac muscle fibres striation (transverse arrow) and pyknotic nuclei(vertical arrow), increased myofibrobast(arrow head) and lymphocytic infiltration(red arrow ) (Hx \& E x 1000).

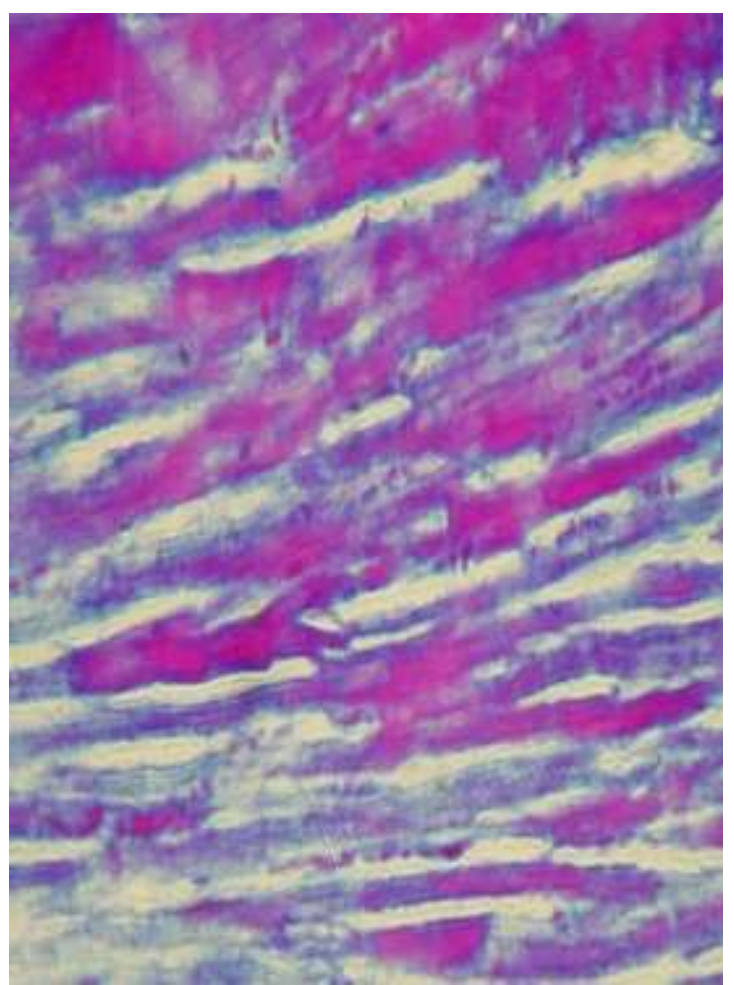

Fig.19: A photomicrograph of cardiac muscle fibres of a rat of group (T1)showing showing mild decrease in collagen fibres between the cardiac muscle fibres (Masson`s trichrome stain, $\mathrm{x} 400$ ).

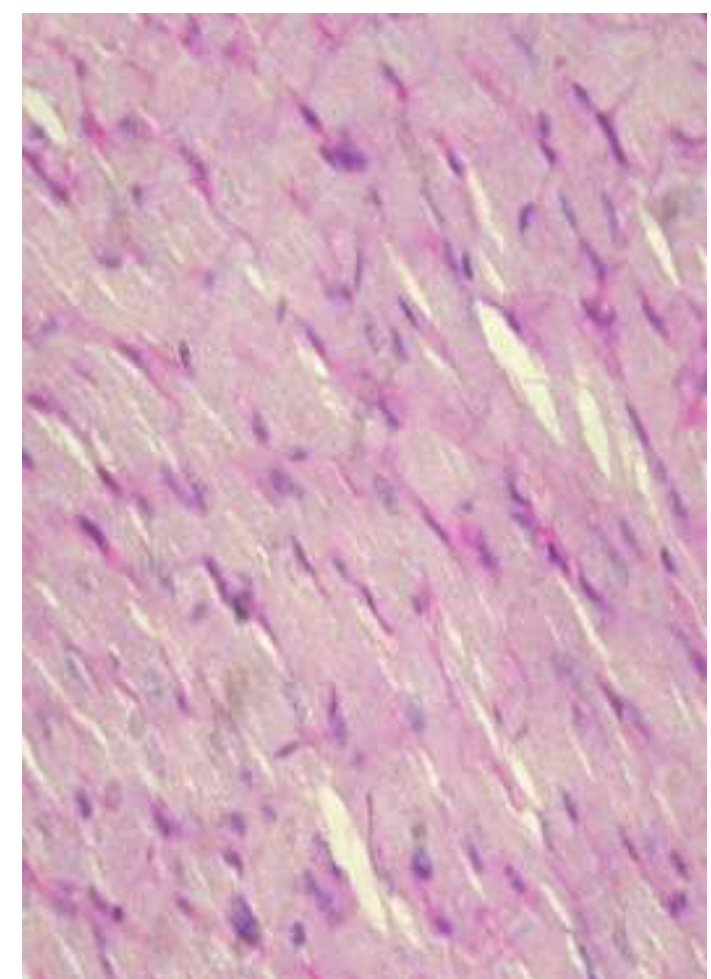

Fig .20: A photomicrograph of cardiac muscle fibres of a rat of group (T1) showing mild increase of PAS + ve materials (magenta red) in the cardiac muscle fibres (PAS reaction $\mathrm{x} 400$ ). 


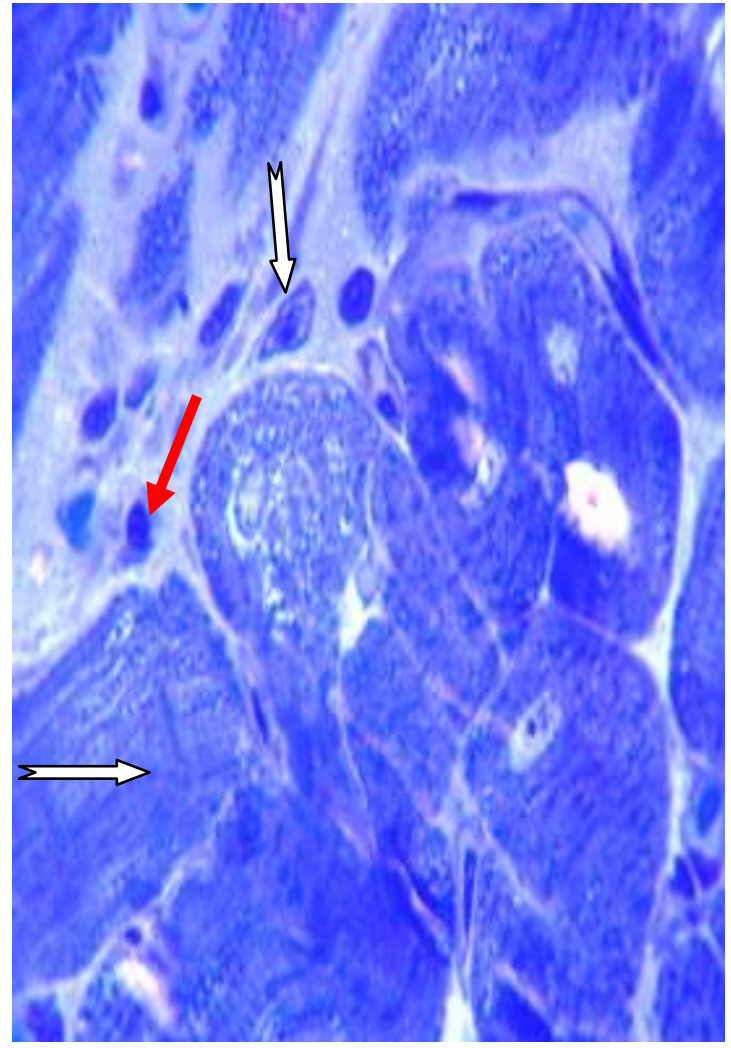

Fig. 21: photomicrograph of cardiac muscle fibres of a rat of group (T1) showing restored striation(transverse arrow) myofibrobast nucleus(vertical arrow)and lymphocytic infiltration

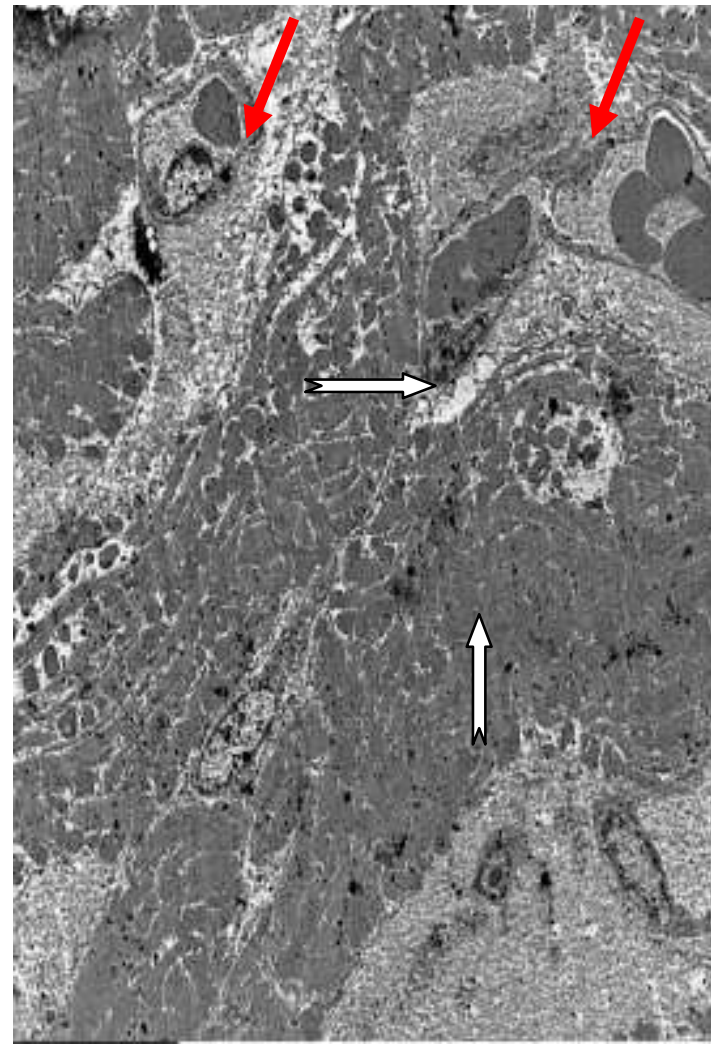

Fig. 22: An electron-micrograph of cardiac muscle fibres of a rat of group (T1) showing nucleus of myofibroblast(transverse arrow) ,congested blood vessels (red arrows) with areas of loss of striation(vertical (semithin section (red arrow),Toluidine blue stain X 1000).

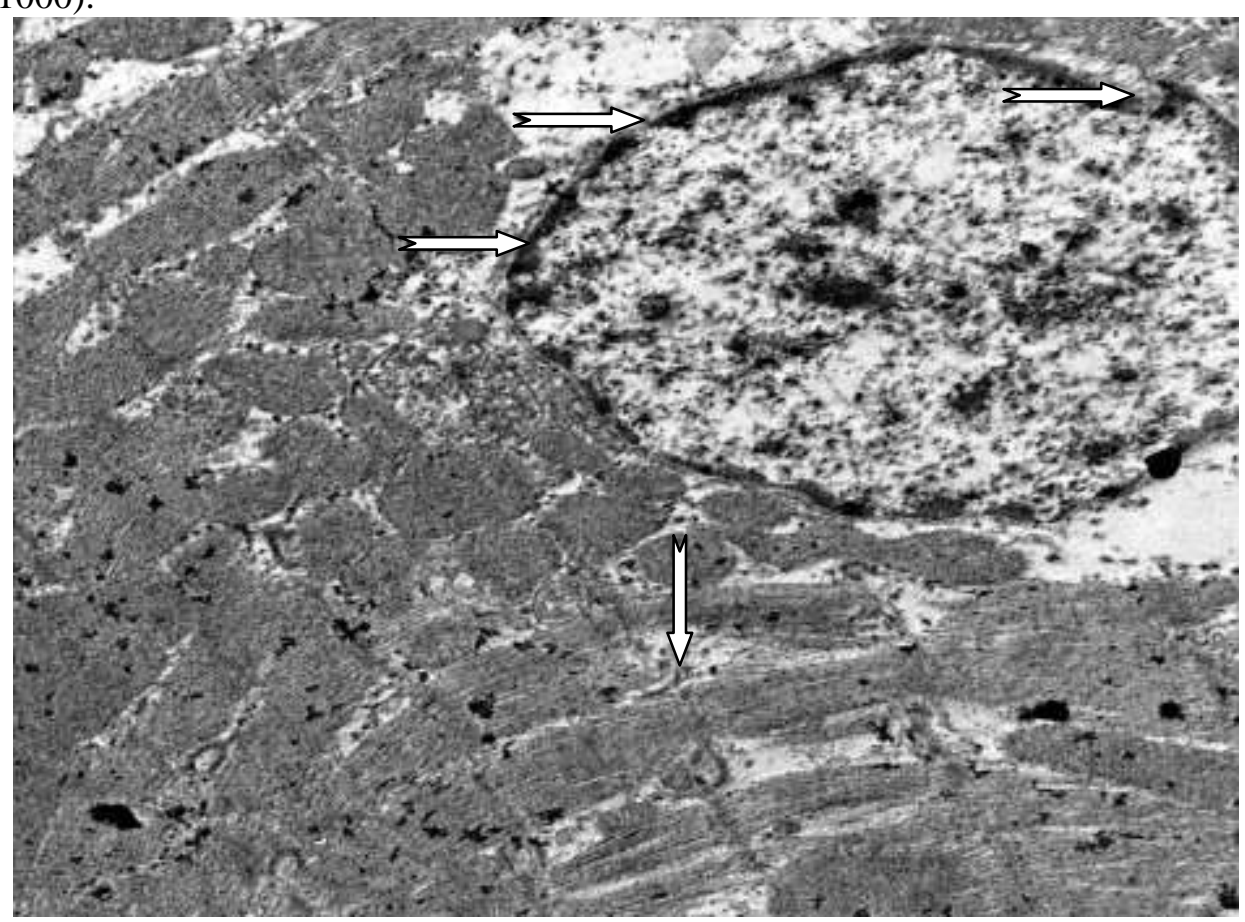

Fig. 23: An electron-micrograph of cardiac muscle fibres of a rat of group (T1) showing vascular nuclear and nuclear pore (transverse arrows) with separated and splitted myofibres(vertical arrow) (X15000). 


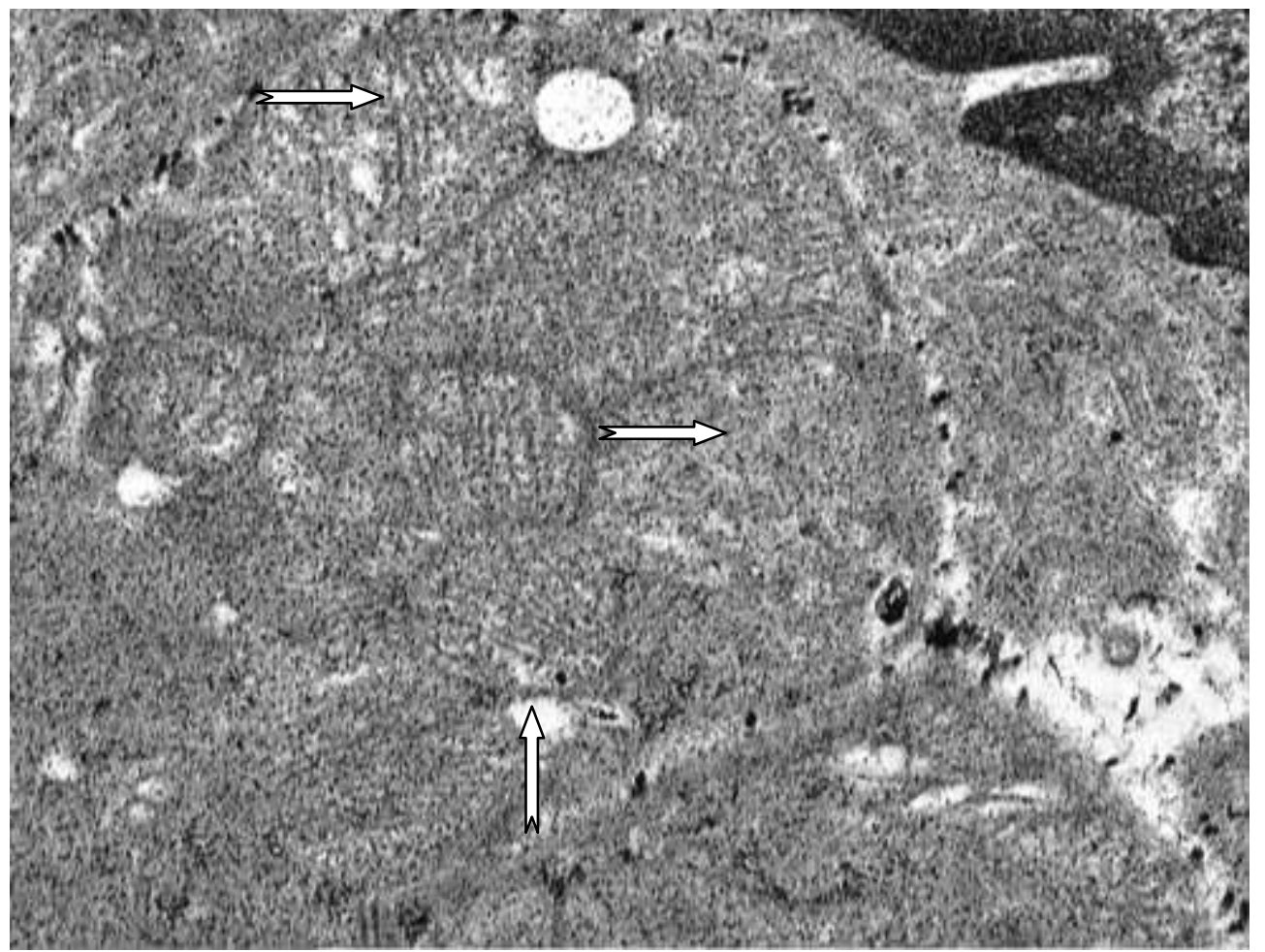

Fig. 24: An electron-micrograph of cardiac muscle fibres of a rat of group (T1) showing mitochondrial vacuolation (vertical arrow) with disorganization of their cristae ( transverse arrow) (X30000).

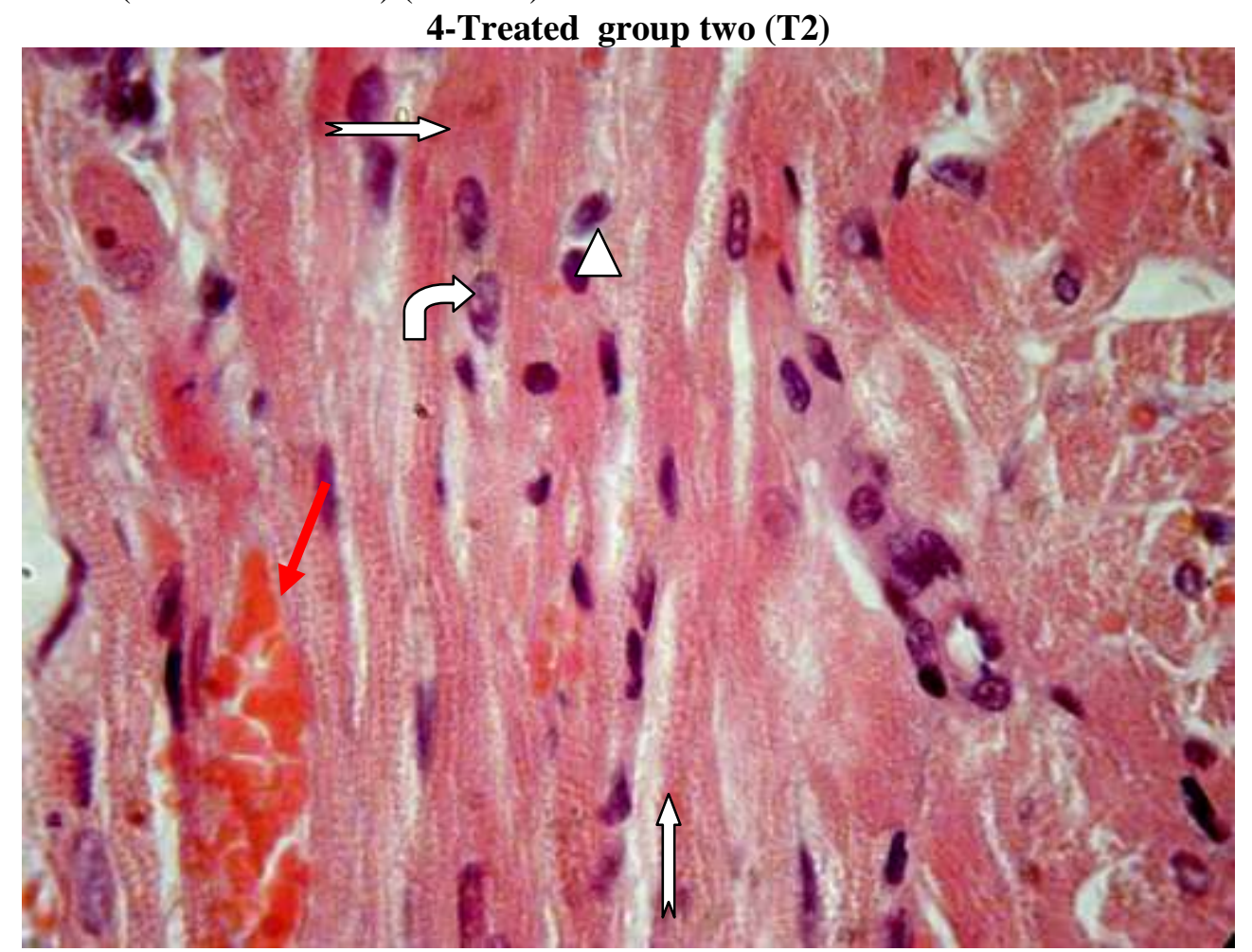

Fig. 25: A photomicrograph of cardiac muscle fibres of a rat of group (T2) showingpatchy loss of striation(transverse arrow) with splitted muscle fibres(vertical arrow), some nuclei become pyknotic(curved arrow) ,congested blood vessel (red arrow) and lymphocyticinfiltration(arrow head) $(\mathrm{Hx} \& \mathrm{E} \times 400)$. 


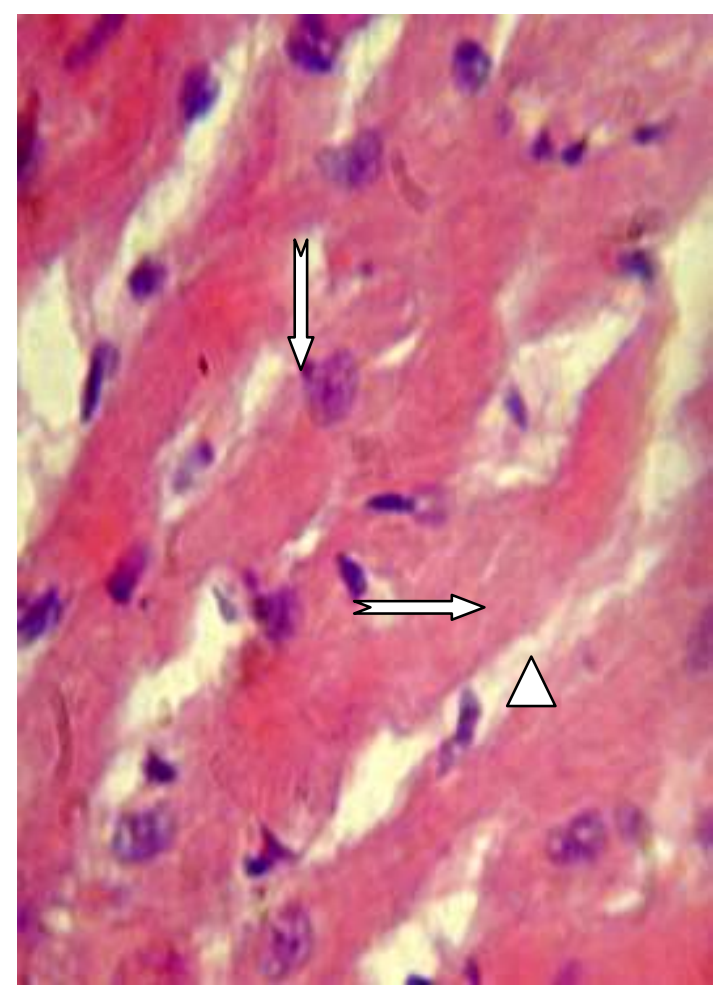

Fig. 26: A higher magnification of the previous section of group (T2) showing patchy loss of striation( transverse arrow) with splitted muscle fibres (arrow head) and pyknotic nucleus with margination of nuclear chromatin (vertical arrow) (Hx\& E x 1000).

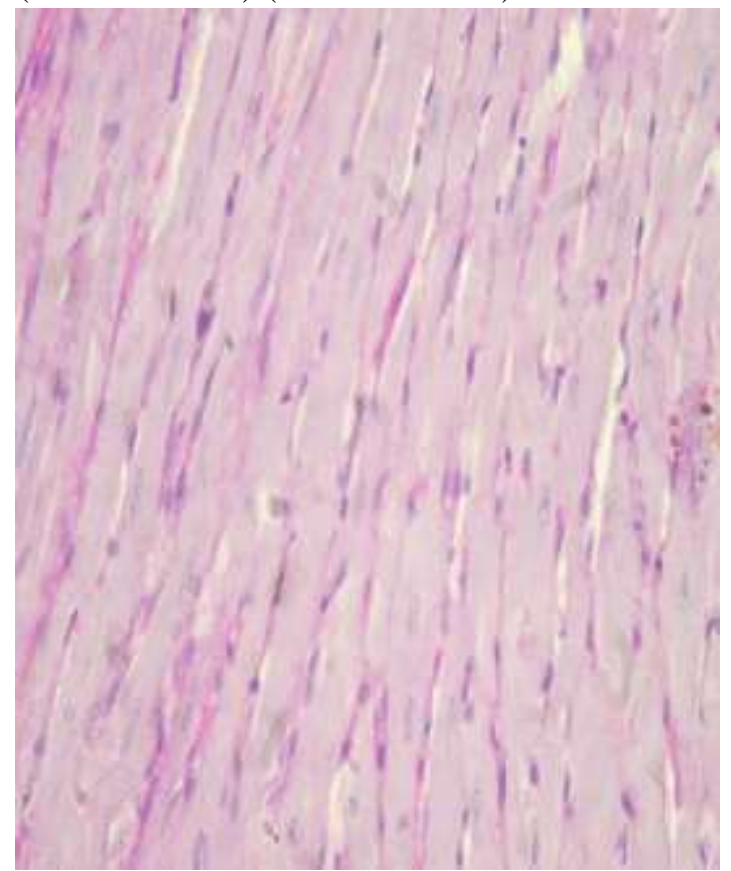

Fig. 28: A photomicrograph of cardiac muscle fibres of a rat of group (T2) showing decreased PAS+ ve materials (magenta red) in the cardiac muscle fibres (PAS reaction, x 400 ).

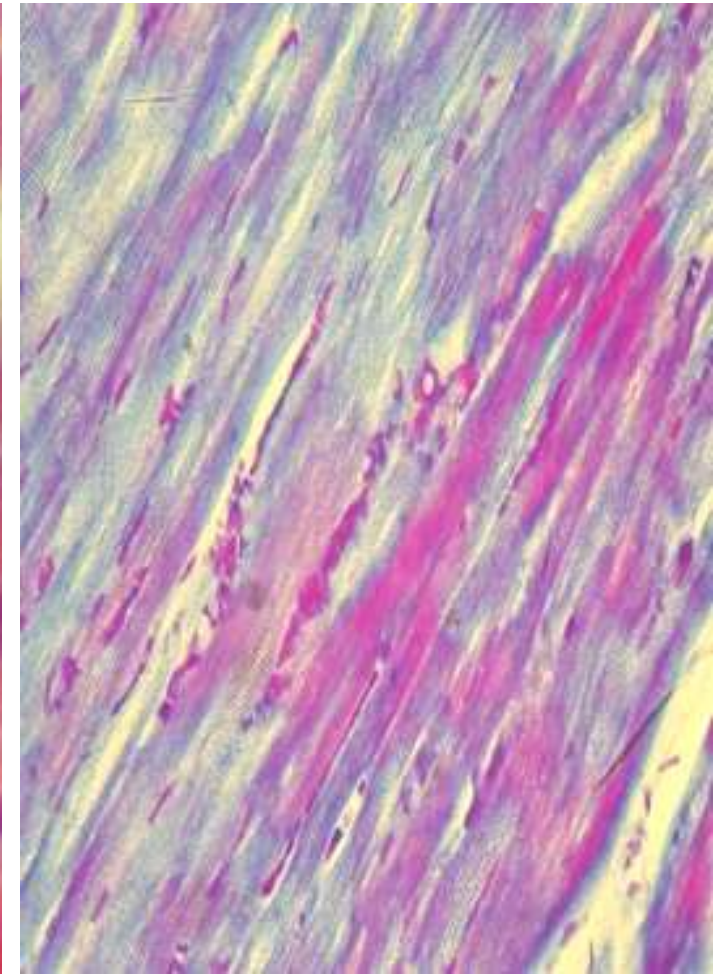

Fig.27:A photomicrograph of cardiac muscle fibres of a rat of group(T2) showing increased collagen fibres between the cardiac muscle fibres .Notice: focal areas of excessive collagen fibres deposition

(Masson`s trichrome stain, x 400 ).

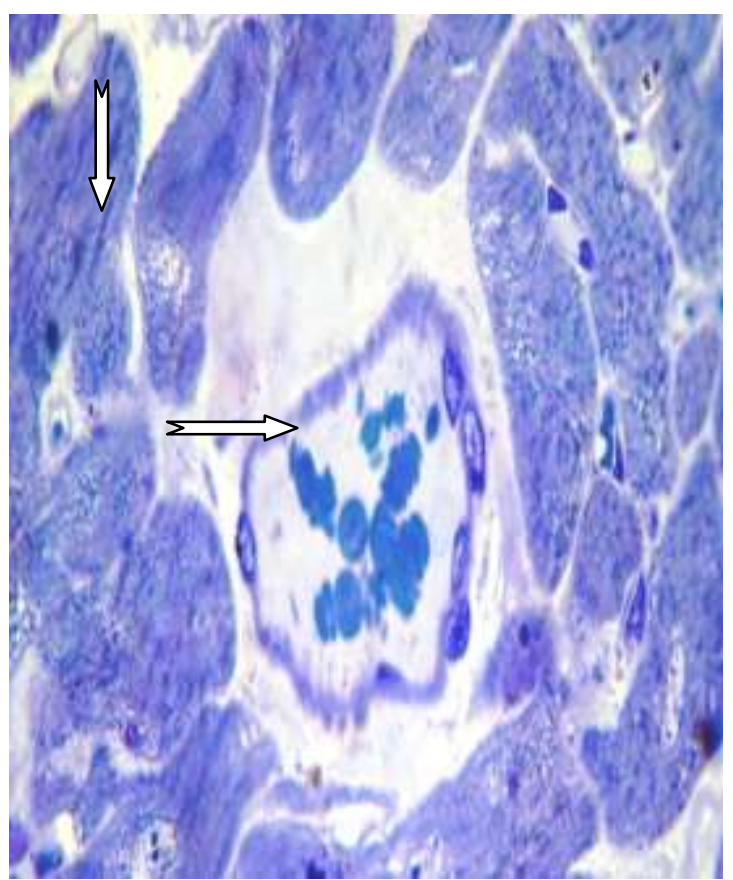

Fig. 29: A photomicrograph of cardiac muscle fibres of a rat of group (T2) showing loss of striation(vertical arrow), dilated and congested blood vessel(transverse arrow) (semithin section, Toluidine blue stain x1000). 


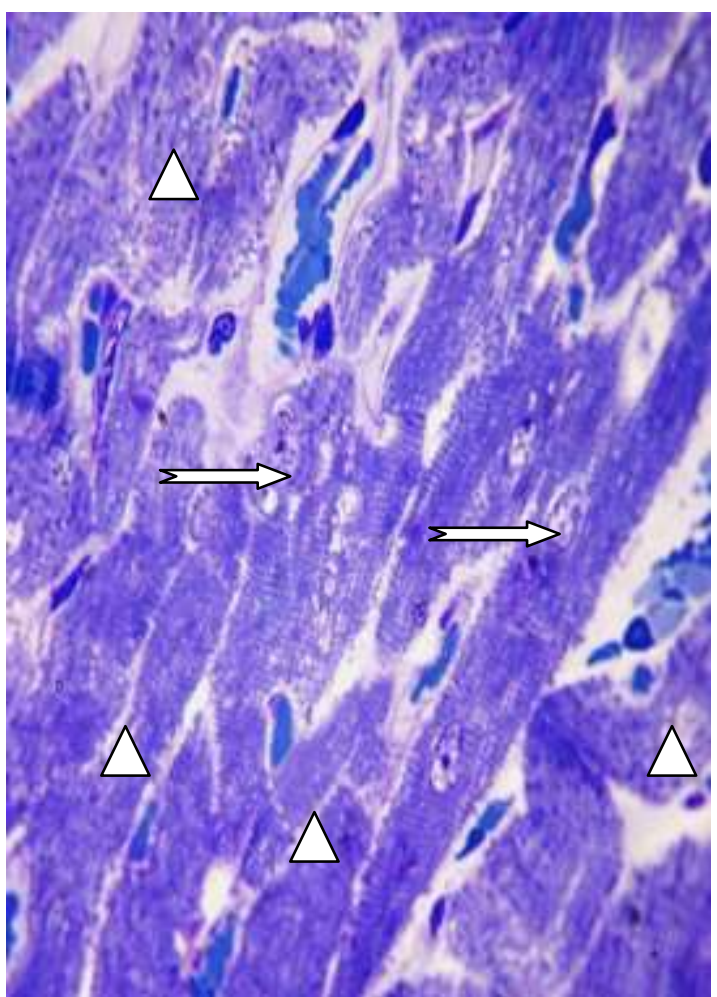

Fig. 30: A photomicrograph of cardiac muscle fibres of a rat of group (T2) showing loss of striation(arrow heads) with vacuolation and nuclear damage (transverse arrows),(semithin section , Toluidine blue stain, $\mathrm{x}$ 1000).

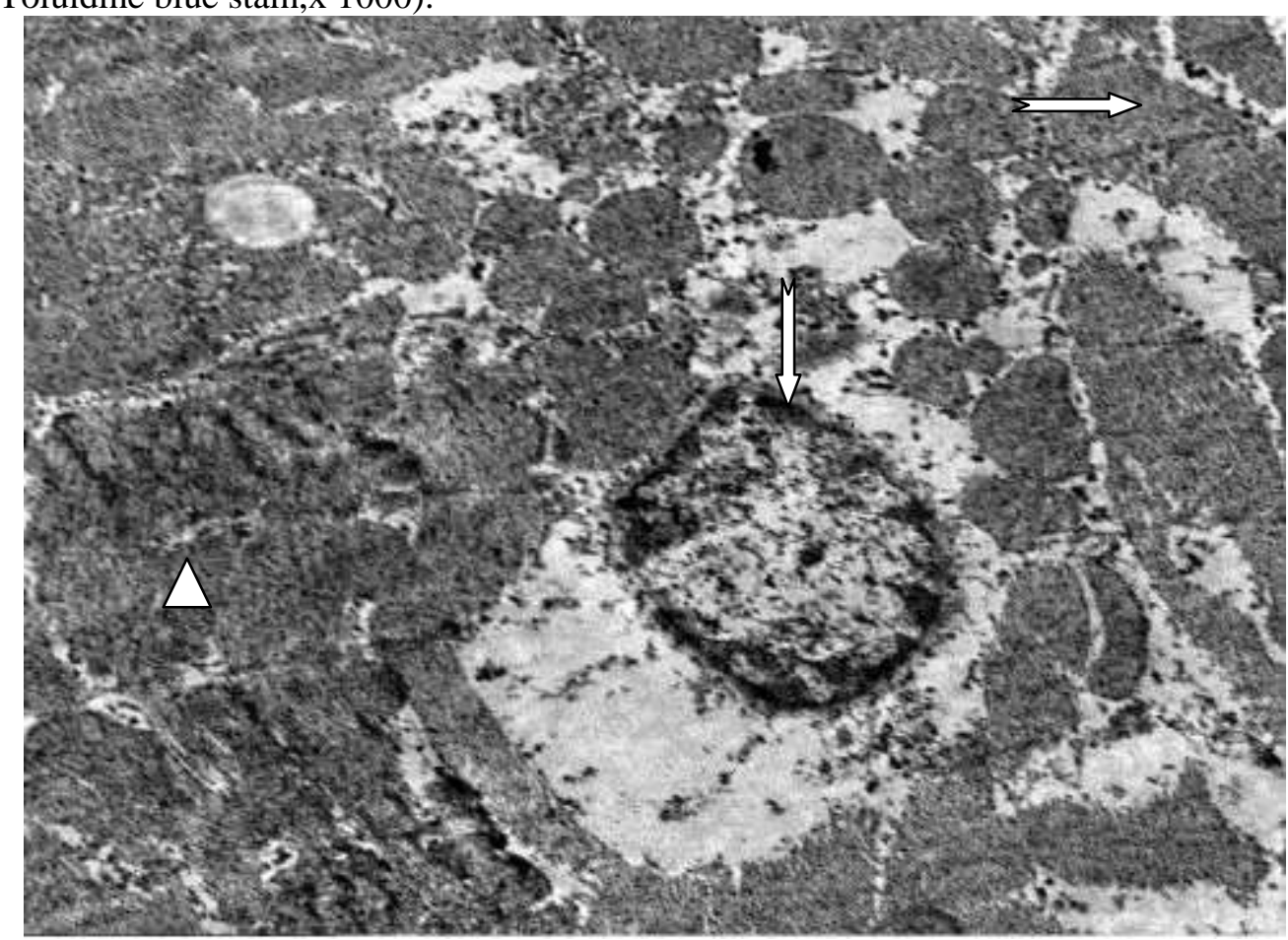

Fig. 32: An electron-micrograph of cardiac muscle fibres of a rat of group

(T2) showing pyknotic nucleus of cardiomyocyte (vertical arrow) with patchy loss of striation (transverse arrow) and disorganization of muscle fibril(arrowhead) (x 15000).

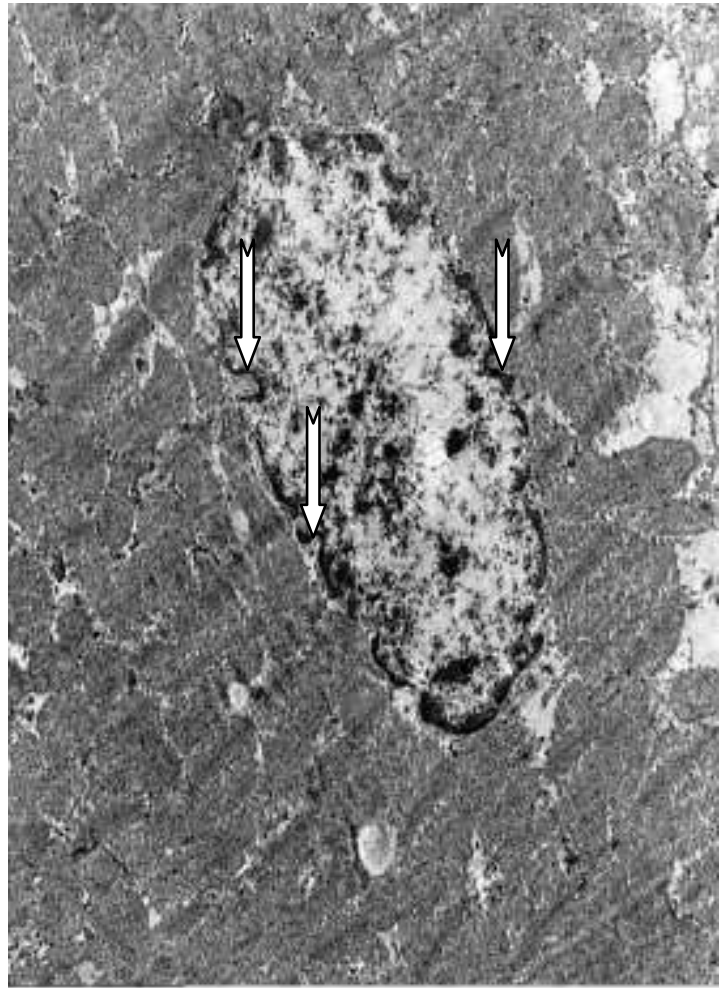

Fig. 31: An electron-micrograph of cardiac muscle fibres of a rat of group (T2) showing indentated degenerated nucleus of cardiomyocytes(vertical arrows) (x15000). 


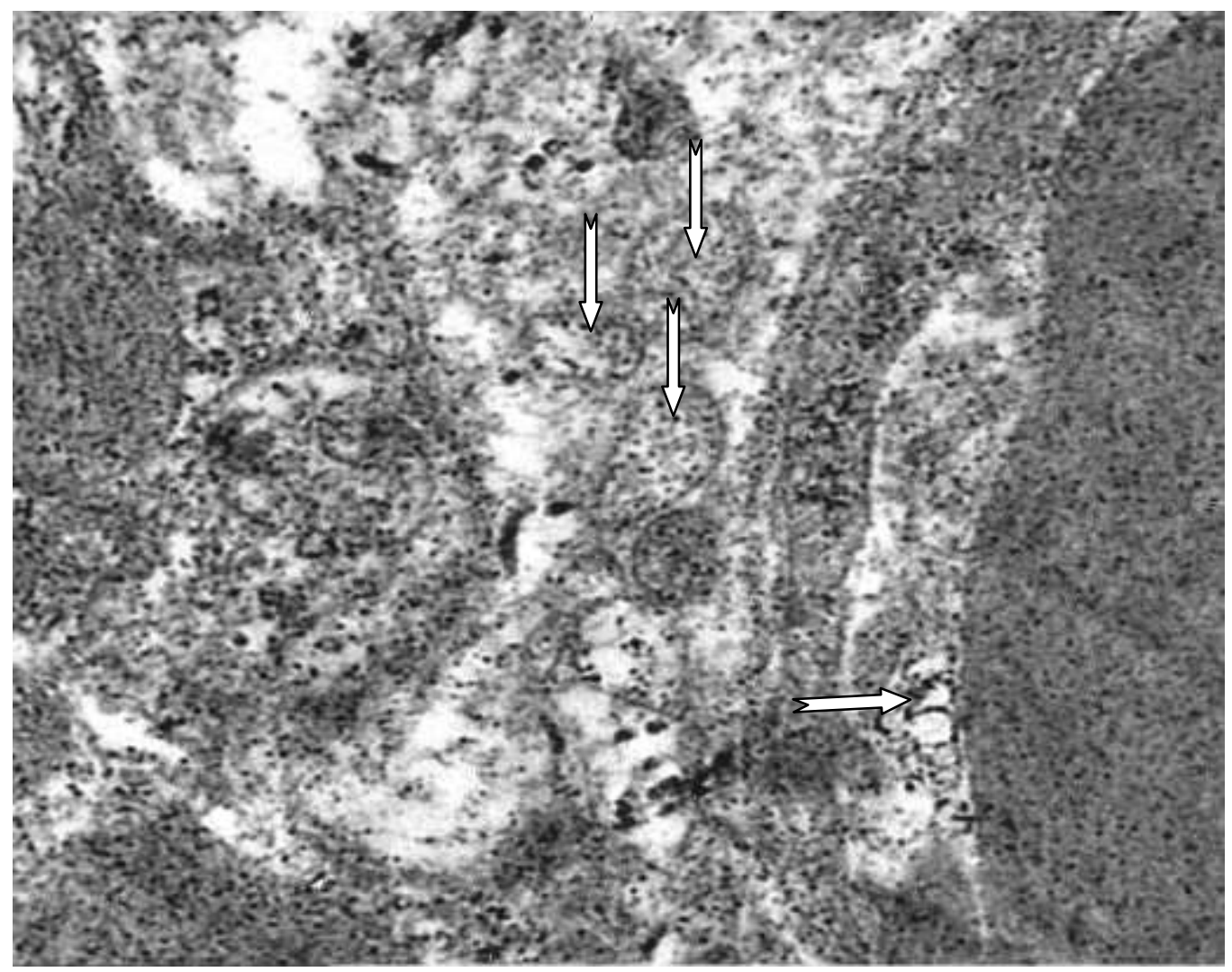

Fig. 33: An electron-micrograph of cardiac muscle fibres of a rat of group (T2) showing disorganization of cristae (vertical arrows) with swelling and destruction of mitochondria (transverse arrows) (X40000).

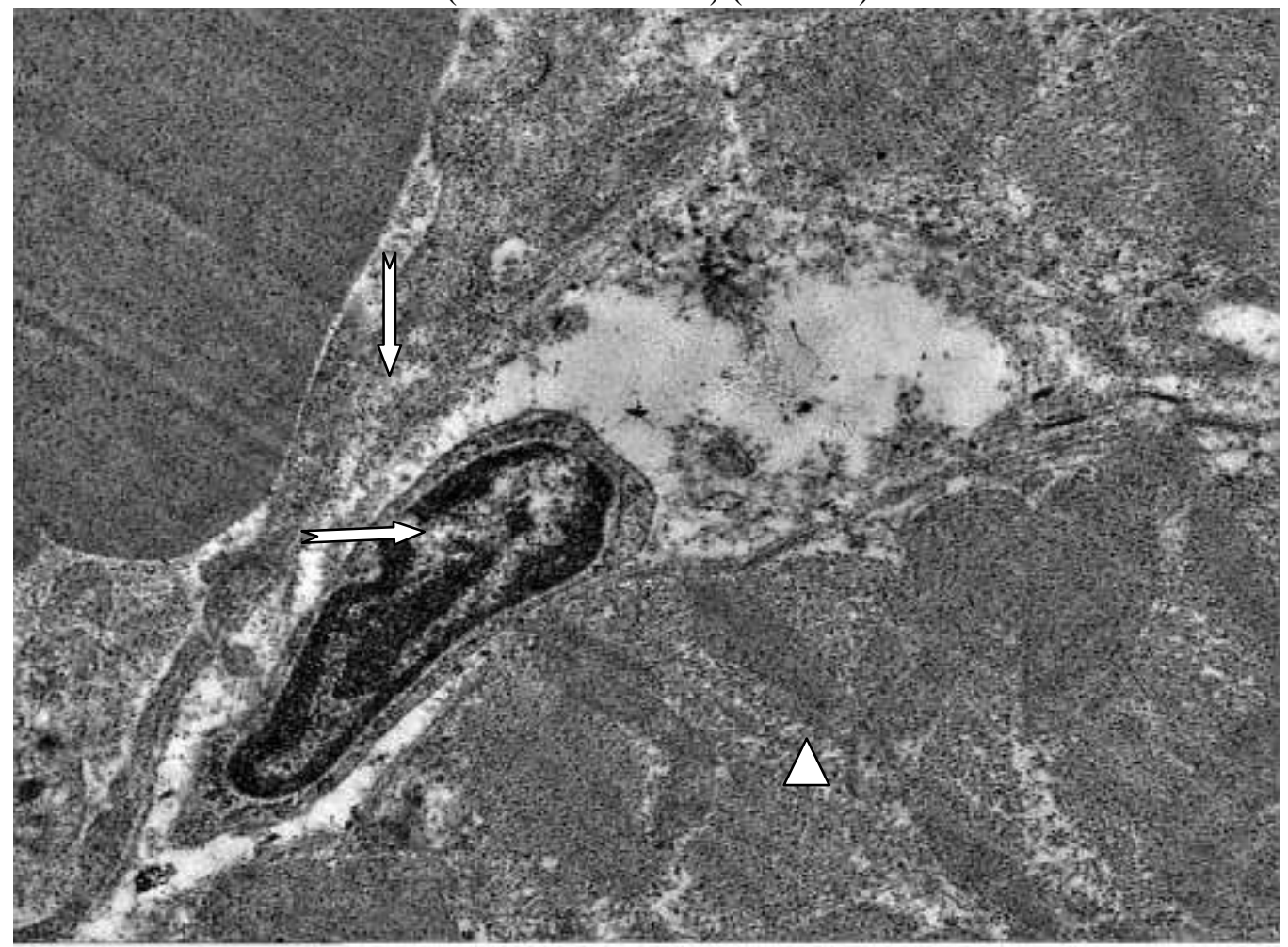

Fig. 34: An electron-micrograph of cardiac muscle fibres of a rat of group (T2)showing nucleus of myofibroblast (transverse arrow) with patchy loss of striation(arrow head) and highly destructed mitochondria with disorganization of their cristae (vertical arrow) (x 20000). 


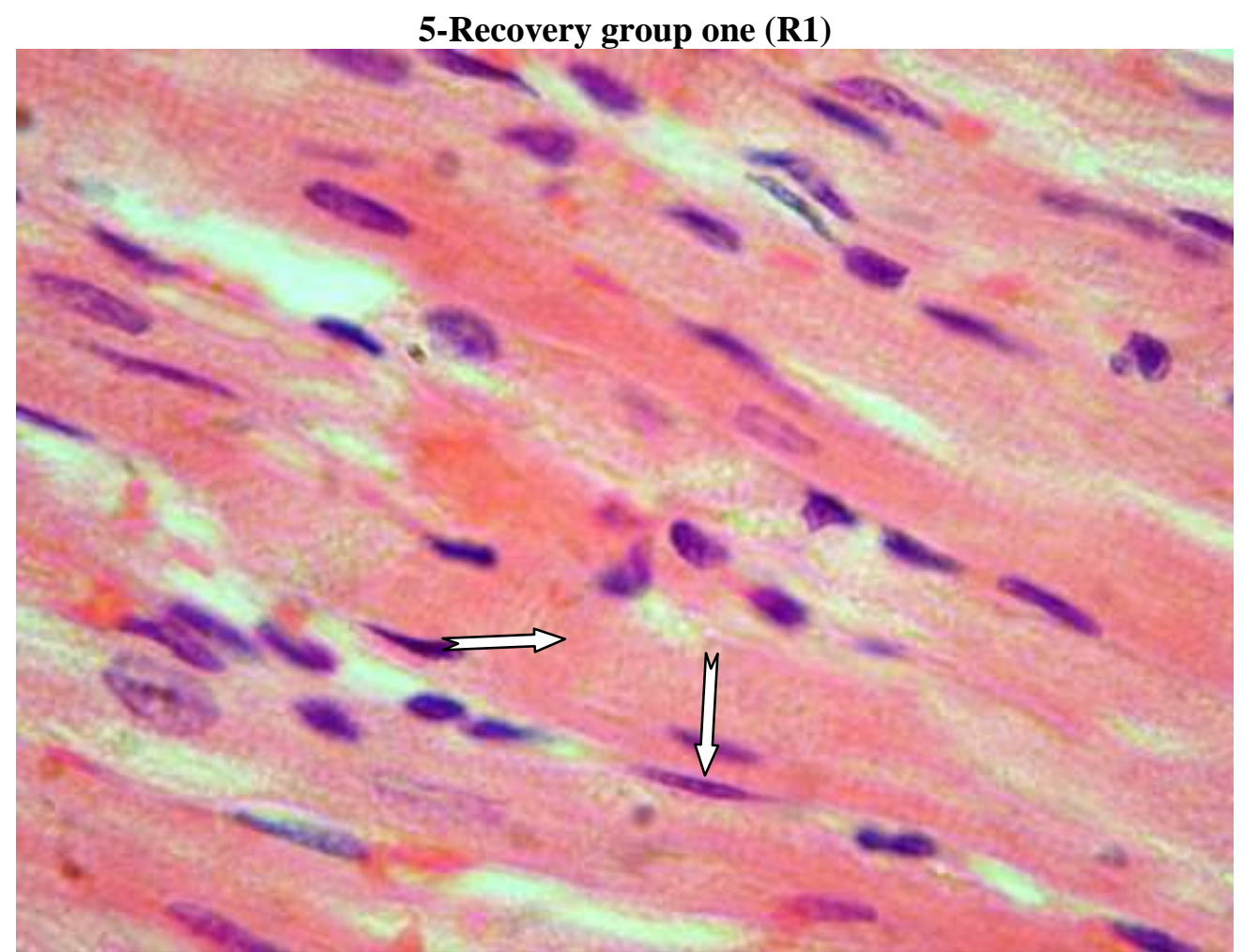

Fig.35 :A photomicrograph of cardiac muscle fibres of a rat of group (R1) showing restored striation(transverse arrow) and myofibroblast(vertical arrow) (H \& E stain $\times 400)$.

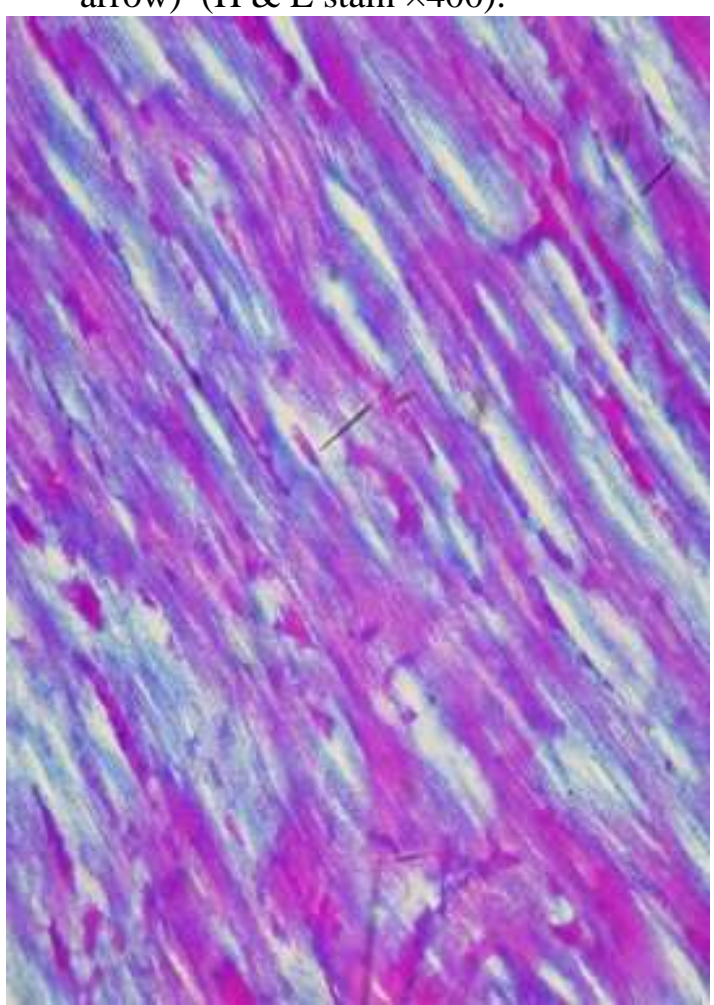

Fig.36: A photomicrograph of cardiac muscle fibres of a rat of group (R1) showing decreased collagen fibres between the cardiac muscle fibres (Masson`s trichrome stain , x 400).

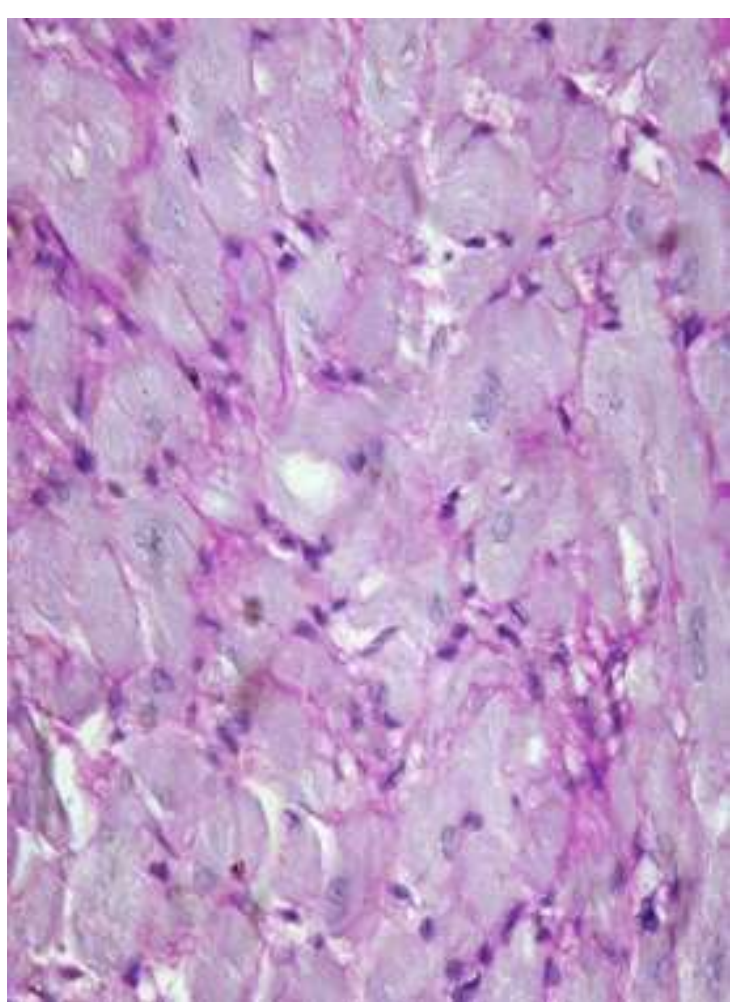

Fig. 37: A photomicrograph of cardiac muscle fibres of a rat of group (R1) showing increased PAS + ve materials (magenta red) in the cardiac fibres (PAS reaction x 400). 


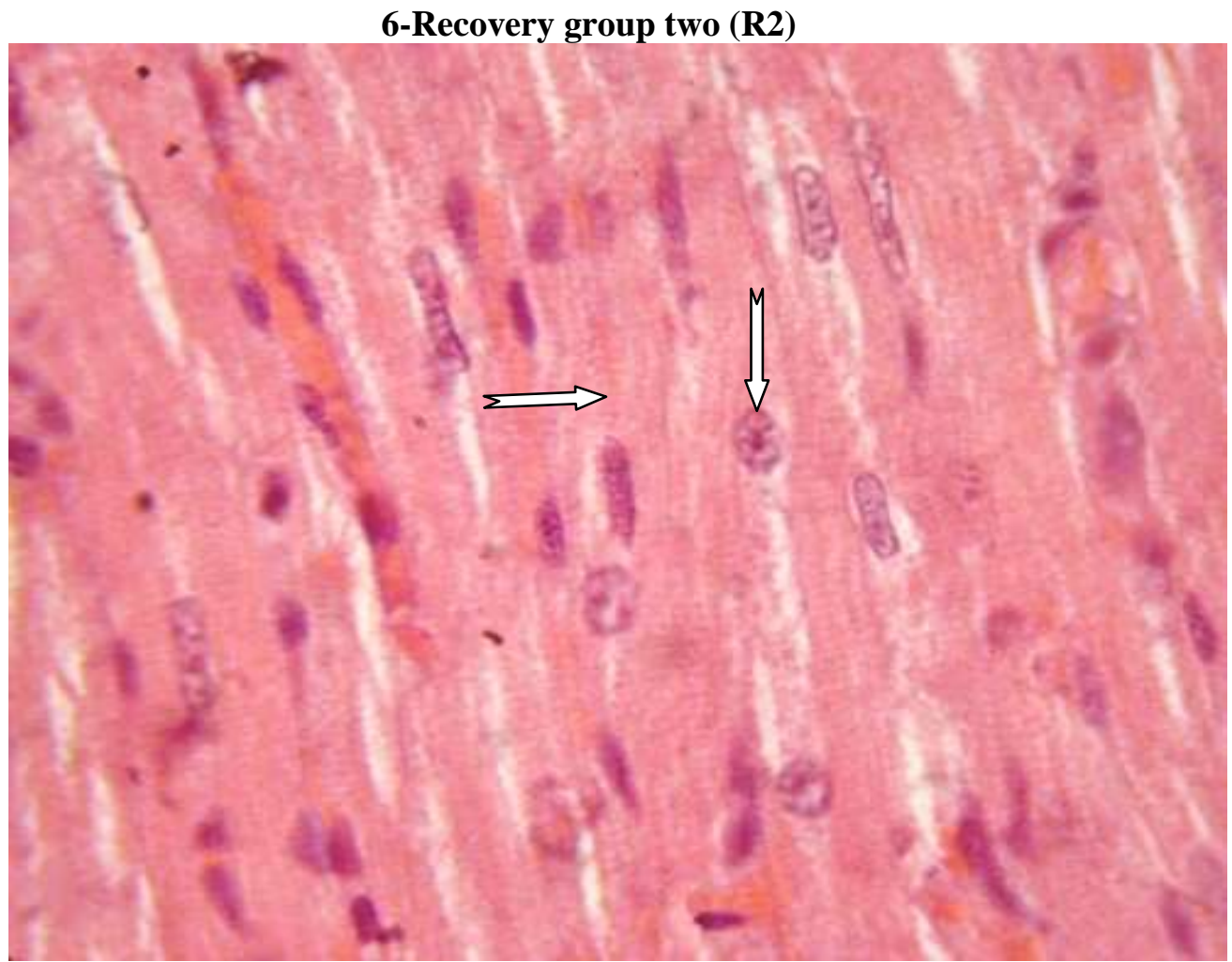

Fig.38: A photomicrograph of cardiac muscle fibres of a rat of group (R 2) showing restored of striation(transverse arrow) and central vesicular nuclei (vertical arrow) of the cardiac muscle fibres $(\mathrm{Hx} \& \mathrm{E} \times 1000)$.

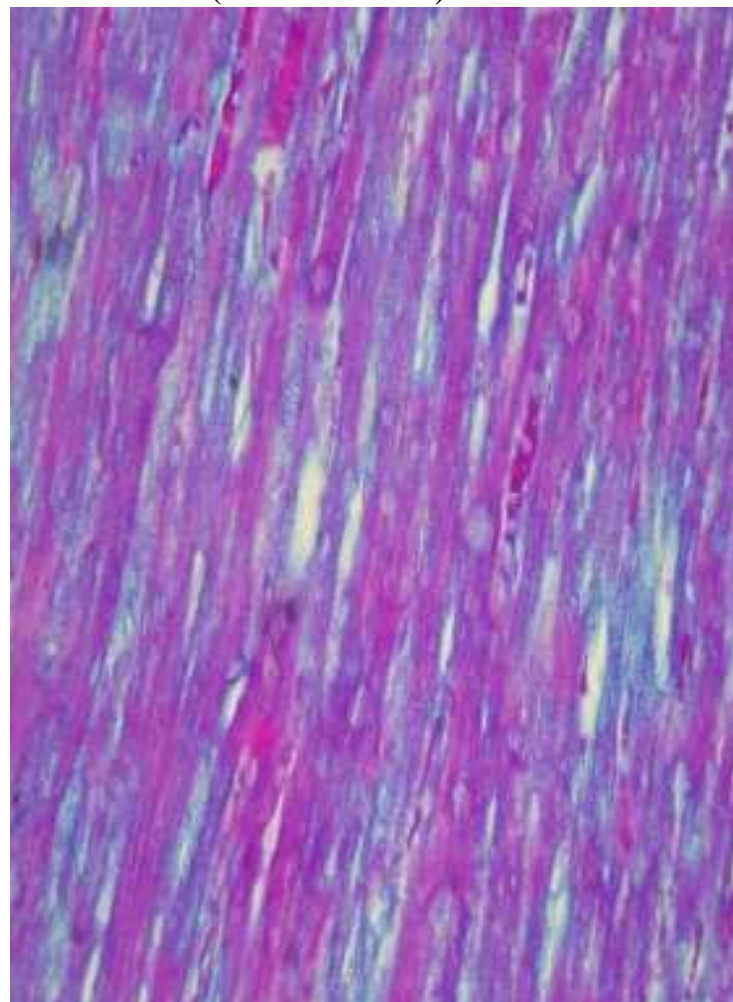

Fig.39: A photomicrograph of cardiac muscle fibres of a rat of group (R 2) showing decreased collagen fibres between the cardiac muscle fibres Masson`s trichrome stain, $\mathrm{x} 400$ ).

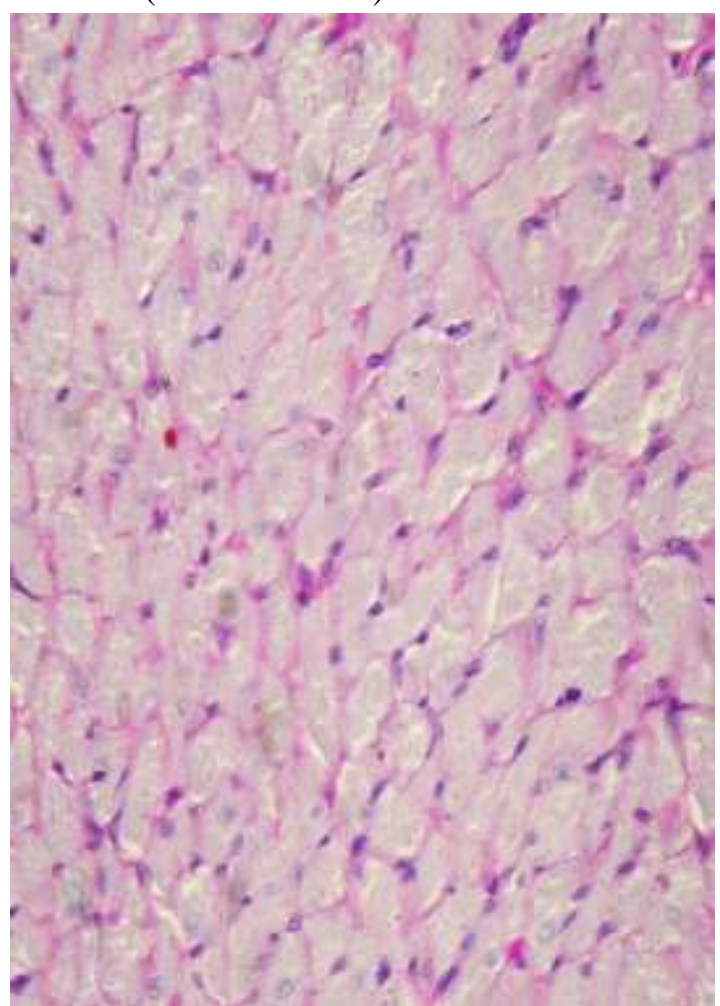

Fig.40: A photomicrograph of cardiac muscle fibres of a rat of group (R 2) showing increased PAS+ ve materials in the cardiac muscle fibers (PAS reaction $\mathrm{x}$ X 400). 
Table. 1: percentage of collagen fibres.

\begin{tabular}{|c|c|c|c|c|c|c|}
\hline & Group I & Group II & Group III & Group IV & Group V & Group VI \\
\cline { 2 - 7 } & control & Hyperlipidemic & Treated 1 & Treated 2 & Recovery 1 & $\begin{array}{c}\text { Recovery } \\
2\end{array}$ \\
\hline Mean & $\mathbf{0 . 9 1}$ & 1.81 & 1.52 & 1.98 & 1.35 & 1.21 \\
\hline N & 10 & 10 & 10 & 10 & 10 & 10 \\
\hline SD \pm & $\mathbf{0 . 1 2}$ & $\mathbf{0 . 3 4}$ & $\mathbf{0 . 2 3}$ & $\mathbf{0 . 4 5}$ & $\mathbf{0 . 1 9}$ & $\mathbf{0 . 1 6}$ \\
\hline Significance & & $\mathbf{p}<\mathbf{0 . 0 5}$ Sig. & $\mathbf{p}<\mathbf{0 . 0 5}$ Sig. & $\mathbf{p}<\mathbf{0 . 0 5}$ Sig. & $\mathbf{p}<\mathbf{0 . 0 5}$ Sig. & $\mathbf{p}<\mathbf{0 . 0 5}$ Sig. \\
\hline
\end{tabular}

Table .2: Optical density of PAS reaction.

\begin{tabular}{|c|c|c|c|c|c|c|}
\hline & Group I & Group II & Group III & Group IV & Group V & Group VI \\
\hline & control & Hyperlipidemic & Treated 1 & Treated 2 & Recovery 1 & Recovery2 \\
\hline Mean & 1.04 & 0.69 & 0.81 & 0.78 & 0.89 & 0.98 \\
\hline $\mathbf{N}$ & 10 & 10 & 10 & 10 & 10 & 10 \\
\hline SD \pm & 0.16 & 0.13 & 0.18 & 0.17 & 0.15 & 0.14 \\
\hline Significance & & $p<0.05$ Sig. & p<0.05 Sig & $p<0.05$ Sig. & p<0.05 Sig. & $p<0.05$ Sig. \\
\hline
\end{tabular}

Table. 3: Cholesterol level.

\begin{tabular}{|c|c|c|c|c|c|c|}
\hline & Group I & Group II & Group III & Group IV & Group V & Group VI \\
\cline { 2 - 7 } & control & Hyperlipidemic & Treated 1 & Treated 2 & Recovery 1 & Recovery2 \\
\hline Mean & $\mathbf{8 4 . 3 7}$ & $\mathbf{1 4 9 . 4 5}$ & $\mathbf{1 2 1 . 7 6}$ & $\mathbf{1 0 2 . 5 1}$ & $\mathbf{9 8 . 6 8}$ & $\mathbf{9 5 . 7 2}$ \\
\hline N & $\mathbf{5}$ & $\mathbf{5}$ & $\mathbf{5}$ & $\mathbf{5}$ & $\mathbf{5}$ & $\mathbf{5}$ \\
\hline SD \pm & $\mathbf{2 . 9 1}$ & $\mathbf{3 . 0 2}$ & $\mathbf{3 . 9 1}$ & $\mathbf{2 . 0 4}$ & $\mathbf{1 . 6 6}$ & $\mathbf{1 . 0 3}$ \\
\hline Significance & \multicolumn{1}{|c}{$<\mathbf{0 . 0 5}$ Sig. } & $\mathbf{p}<\mathbf{0 . 0 5}$ Sig. & $\mathbf{p}<\mathbf{0 . 0 5}$ Sig. & $\mathbf{p}<\mathbf{0 . 0 5}$ Sig. & p $<\mathbf{0 . 0 5}$ Sig. \\
\hline
\end{tabular}

Table. 4:Triglycerides level.

\begin{tabular}{|c|c|c|c|c|c|c|}
\hline & Group I & Group II & Group III & Group IV & Group V & Group VI \\
\cline { 2 - 7 } & control & Hyperlipidemic & Treated 1 & Treated 2 & Recovery 1 & Recovery2 \\
\hline Mean & 90.62 & 158.85 & 134.20 & 115.10 & 105.73 & 98.73 \\
\hline N & 5 & 5 & 5 & 5 & 5 & 5 \\
\hline SD \pm & 1.83 & 4.81 & 3.18 & 2.59 & 2.67 & 1.98 \\
\hline Significance & & $\mathbf{p}<0.05$ Sig. & p<0.05 Sig. & p<0.05 Sig. & p<0.05 Sig. & p<0.05 Sig. \\
\hline
\end{tabular}


Table. 5: LDL-C level.

\begin{tabular}{|c|c|c|c|c|c|c|}
\hline & Group I & Group II & Group III & Group IV & Group V & Group VI \\
\hline & control & Hyperlipidemic & Treated 1 & Treated 2 & Recovery 1 & Recovery 2 \\
\hline Mean & 38.33 & 99.03 & 72.77 & 55.90 & 52.81 & 49.70 \\
\hline $\mathbf{N}$ & 5 & 5 & 5 & 5 & 5 & 5 \\
\hline SD \pm & 0.05 & 1.28 & 1.12 & 1.03 & 0.97 & 0.78 \\
\hline Significance & & p<0.05 Sig. & $p<0.05$ Sig & $p<0.05$ Sig. & $p<0.05$ Sig. & $p<0.05$ Sig. \\
\hline
\end{tabular}

Table. 6: HDL-C level.

\begin{tabular}{|c|c|c|c|c|c|c|}
\hline & Group I & Group II & Group III & Group IV & Group V & Group VI \\
\cline { 2 - 7 } & control & Hyperlipidemic & Treated 1 & Treated 2 & Recovery 1 & Recovery2 \\
\hline Mean & 27.91 & 18.65 & 21.90 & 23 & 24.73 & 26.29 \\
\hline N & 5 & 5 & 5 & 5 & 5 & 5 \\
\hline SD \pm & $\mathbf{0 . 9 9}$ & 1.14 & 1.08 & $\mathbf{0 . 7 8}$ & $\mathbf{0 . 5 4}$ & $\mathbf{0 . 7 5}$ \\
\hline Significance & & $\mathbf{p}<\mathbf{0 . 0 5}$ Sig. & $\mathbf{p}<0.05$ Sig.p $<0.05$ Sig. & $\mathbf{p}<0.05$ Sig. & $\mathbf{p}<0.05$ Sig. \\
\hline
\end{tabular}

Table .7 : VLDL

\begin{tabular}{|c|c|c|c|c|c|c|}
\hline & Group I & Group II & Group III & Group IV & Group V & Group VI \\
\cline { 2 - 7 } & control & Hyperlipidemic & Treated 1 & Treated 2 & Recovery 1 & Recovery2 \\
\hline Mean & 18.12 & 31.77 & 26.84 & 23.02 & 21.14 & 19.74 \\
\hline N & 5 & 5 & 5 & 5 & 5 & 5 \\
\hline SD \pm & $\mathbf{0 . 8 6}$ & 1.18 & 1.20 & 0.99 & 0.93 & 0.9 \\
\hline Significance & & p $<0.05$ Sig. & p<0.05 Sig.p $\mathbf{p}<0.05$ Sig. & $\mathbf{p}<0.05$ Sig. & p $<0.05$ Sig. \\
\hline
\end{tabular}

Table .8 :Atherogenic index (AI)

\begin{tabular}{|c|c|c|c|c|c|c|}
\hline & Group I & Group II & Group III & Group IV & Group V & Group VI \\
\cline { 2 - 7 } & control & Hyperlipidemic & Treated 1 & Treated 2 & Recovery 1 & Recovery2 \\
\hline Mean & $\mathbf{3 . 2 4}$ & $\mathbf{8 . 5 1}$ & 6.12 & 5 & 4.27 & 3.64 \\
\hline N & 5 & 5 & 5 & 5 & 5 & 5 \\
\hline SD \pm & $\mathbf{0 . 6 1}$ & $\mathbf{1 . 2 5}$ & $\mathbf{0 . 9 3}$ & $\mathbf{1 . 5 8}$ & $\mathbf{0 . 7 9}$ & $\mathbf{0 . 7 2}$ \\
\hline Significance & & $\mathbf{p}<\mathbf{0 . 0 5}$ Sig. & $\mathbf{p}<\mathbf{0 . 0 5}$ Sig.p $\mathbf{p}<0.05$ Sig. & $\mathbf{p}<0.05$ Sig. & p<0.05 Sig. \\
\hline
\end{tabular}

